\title{
Light scattering from random rough dielectric surfaces
}

\author{
J. A. Sánchez-Gil and M. Nieto-Vesperinas \\ Instituto de Optica, Consejo Superior de Investigaciones Científicas, Serrano 121, Madrid 28006, Spain
}

\begin{abstract}
Received July 9, 1990; revised manuscript received January 31, 1991; accepted March 12, 1991
A theoretical and numerical study is made of the scattering of light and other electromagnetic waves from rough surfaces separating vacuum from a dielectric. The extinction theorem, both above and below the surface, is used to obtain the boundary values of the field and its normal derivative. Then we calculate the angular distribution of the ensemble average of intensity of the reflected and transmitted fields. The scattering equations are solved numerically by generating one-dimensional surface profiles through a Monte Carlo method. The effect of roughness $\sigma$ and correlation distance $T$ on the aforementioned angular distribution, as well as on the reflectance, is analyzed. Enhanced backscattering and new transmission effects are observed, also depending on the permittivity. The ratio $\sigma / T$ is large in all cases studied, and thus no analytical approximation, such as the Kirchhoff approximation (KA) and small perturbation methods, could a priori be expected to hold. We find, however, that the range of validity of the KA can be much broader than that previously found in perfect conductors.
\end{abstract}

\section{INTRODUCTION}

The scattering of light and other electromagnetic waves from rough surfaces is a subject of broad interest. Since the prediction of polariton localization, ${ }^{1,2}$ the appearance of experimental results with rough surfaces of controlled statistics, ${ }^{3,4}$ and the report of new numerical methods of calculating scattering equations, ${ }^{5-8}$ there has been renewed activity in both experimental and theoretical research. ${ }^{9-20}$ In general, the phenomena studied in these papers, such as enhanced backscattering, blaze, quasiLambertian scattering, and forward scattering, are due to multiple scattering in rather high corrugations. The numerical procedures enable us to obtain new results that are not accounted for by the analytical approximations formerly used, namely, the Kirchhoff approximation ${ }^{21-23}$ (KA) and the small perturbation method. ${ }^{24-31}$

Scattering equations, based on the extinction theorem ${ }^{32}$ (ET), are solved numerically. This procedure was initially applied to perfect conductors ${ }^{5,6}$ and later extended to real metals and dielectrics. ${ }^{7,8}$ On the other hand, recent new experiments with metal and dielectric surfaces have yielded interesting results concerning the angular distribution of diffusely reflected light,, 910 and it is important to test the theory with those experiments as well as to predict new effects.

In the present paper we include a detailed study of scattering from both shallow and deep interfaces separating vacuum from a lossless dielectric medium $\{\mathfrak{N}[\epsilon(\omega)]>0$, $\Im[\epsilon(\omega)] \simeq 0, \mathfrak{N}$ and $\Im$ denoting real and imaginary parts, respectively, and $\epsilon(\omega)$ being the dielectric permittivity\}. For the angular distribution of mean reflected and transmitted intensities for $s$ and $p$ polarization, a numerical method based on the ET is developed (we choose the convention $s$ and $p$ for TE and TM waves, respectively). This method is similar to that of Refs. 7 and 8, the difference pertaining to the incident field, which here is assumed to be a plane wave of wavelength $\lambda$ instead of a Gaussian beam. Moreover, new results for the reflected and transmitted fields are obtained here. In addition, the re- flectance (total normalized reflected energy) and the transmittance (total normalized transmitted energy) are calculated and show the influence of roughness on these parameters. The unitarity condition, which should hold when the reflectance and the transmittance are added, is used as a criterion of numerical consistency of the method.

We have also worked with the KA. ${ }^{21-23}$ With the use of this simpler method, the physical nature of the phenomena involved in both reflection and transmission is analyzed in some cases. Concerning light transmission, the recent theoretical predictions, supported by experimental measures (both results are shown in Ref. 19), are submitted here to deeper research. We confirm the conjecture of Ref. 19, according to which the range of validity of the $\mathrm{KA}$ is much broader for dielectrics than was previously found for perfect conductors. ${ }^{6}$

The one-dimensional random surfaces, which are generated by the Monte Carlo method ${ }^{5-8,33}$ as outlined in Subsection 2.D below, possess a known Gaussian power spectrum characterized by the rms height $\sigma$ and the correlation length $T$ of the random height. The angular distribution of the mean scattered intensity is calculated from the average over several surface samples of length $L$. Several angles of incidence $\theta_{o}$ and surface parameters $\sigma$ and $T$ are considered. From those distributions, both the reflectance and the transmittance are derived, and, consequently, so is the unitarity condition. Apart from the one dimensionality of the surfaces, from which we cannot predict cross-polarized scattering (i.e., $s p$ or $p s$ ), there are two other limitations in our results: first, the finite number of sampling points and the consequent finite length of the illuminated surface $L$; second, the limited number of samples over which we perform the ensemble average of the angular distribution of scattered intensities.

This paper is organized as follows: In order to describe the method clearly, we present the scattering equations in Section 2; then the KA method is addressed for a dielectric interface. The numerical expressions for those equations are written at the end of Section 2 in a simple formalism. The numerical solutions obtained with the 


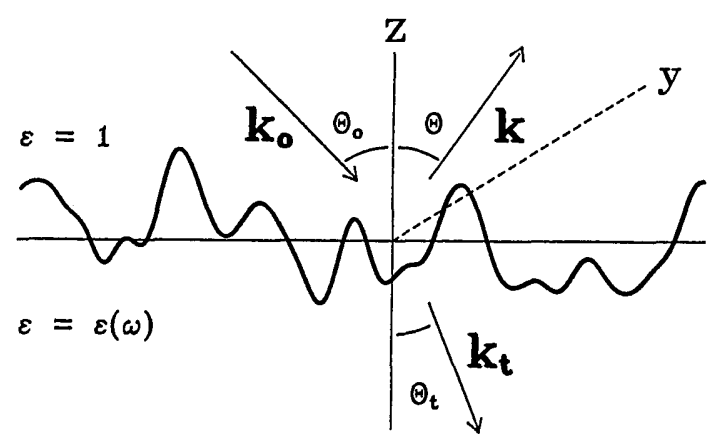

Fig. 1. Scattering geometry.

ET at two different regimes of $T$, namely, $T>\lambda$ and $T<\lambda$, are addressed in Section 3. Section 3 also contains the predictions of the KA. In Section 4 a general discussion of the results is given, leading to several conclusions, which are stated in Section 5.

\section{THEORY}

\section{A. Scattering Equations}

The physical system considered is shown in Fig. 1. It consists of a rough interface $z=D(x)$ that depends only on the $x$ coordinate and separates a semi-infinite vacuum $\tilde{V}[z>D(x)]$ from a semi-infinite dielectric medium $V[z<$ $D(x)]$ characterized by a linear, spatially uniform and isotropic, frequency-dependent dielectric constant $\epsilon(\omega)$.

A linearly polarized monochromatic plane electromagnetic wave is incident from vacuum upon the surface at an angle $\theta_{o}$ with the $z$ axis. The components of the incident, reflected, and transmitted wave vectors are, respectively,

$$
\begin{aligned}
\mathbf{K}_{o} & \equiv k_{o}\left(\sin \theta_{o}, 0,-\cos \theta_{o}\right), \\
\mathbf{K} & \equiv k_{o}(\sin \theta, 0, \cos \theta), \\
\mathbf{K}_{t} & \equiv[\epsilon(\omega)]^{1 / 2} k_{o}\left(\sin \theta_{t}, 0,-\cos \theta_{t}\right),
\end{aligned}
$$

and their moduli hold:

$$
\begin{aligned}
|\mathbf{K}|^{2} & =\left|\mathbf{K}_{o}\right|^{2}=k_{o}{ }^{2}=\frac{\omega^{2}}{c^{2}}=(2 \pi / \lambda)^{2}, \\
\left|\mathbf{K}_{t}\right|^{2} & =\epsilon(\omega) k_{o}{ }^{2},
\end{aligned}
$$

$\lambda$ being the wavelength of the incident plane wave. Since the surface variation occurs in the $x$ coordinate only, there is no depolarization for either $s$ or $p$ incident waves; i.e., we can restrict the analysis in these cases to that of a scalar problem. Thus the electric field for $s$ polarization (TE waves) and the magnetic field for $p$ polarization (TM waves) have just one nonzero component: the $y$ component.

For $s$ polarization, the incident electric vector is written as

$$
\mathbf{E}^{(i)}(\mathbf{r})=\hat{j} E^{(i)} \exp \left(i \mathbf{K}_{o} \cdot \mathbf{r}\right) .
$$

Analogously, for $p$ polarization, the incident magnetic vector is expressed as

$$
\mathbf{H}^{(i)}(\mathbf{r})=\hat{j} H^{(i)} \exp \left(i \mathbf{K}_{o} \cdot \mathbf{r}\right),
$$

where $\mathbf{r}=(x, z), \hat{j}$ is the unit vector along $O Y$, and $E^{(i)}$ and $H^{(i)}$ are complex constant amplitudes. A time-dependence factor $\exp (-i \omega t)$ is suppressed everywhere, as is the $y$ dependence of the vectors $\mathbf{r}, \mathbf{K}_{o}, \mathbf{K}$, and $\mathbf{K}_{t}$.

The scattered fields above the surface (reflected) and below the surface transmitted, only if $\mathfrak{R}[\epsilon(\omega)]>0\}$ are derived by our solving the corresponding Helmholtz equation. In what follows we study each polarization separately.

\section{1. s Polarization}

In order to find the electric field, we must solve the following pair of Helmholtz equations:

$$
\begin{aligned}
& \nabla^{2} E^{\text {(out) }}(\mathbf{r})+k_{o}{ }^{2} E^{\text {(out) }}(\mathbf{r})=0, \quad z>D(x),(\mathbf{r} \in \tilde{V}), \\
& \nabla^{2} E^{(\mathrm{in})}(\mathbf{r})+\epsilon(\omega) k_{o}{ }^{2} E^{(\mathrm{in})}(\mathbf{r})=0, \quad z<D(x),(\mathbf{r} \in V) .
\end{aligned}
$$

The superscripts (out) and (in) mean inside vacuum and dielectric, respectively, and $E^{\text {(out) }}(\mathbf{r})$ and $E^{(\text {in) }}(\mathbf{r})$ denote the complex amplitudes of the electric vectors, which have only a $y$ Cartesian component.

The continuity conditions

$$
\begin{gathered}
{\left[\mathbf{E}^{(\text {in) }}-\mathbf{E}^{(\text {out })}\right] \times \hat{n}=0,} \\
{\left[\mathbf{H}^{(\text {in) }}-\mathbf{H}^{\text {(out) }}\right] \times \hat{n}=0,}
\end{gathered}
$$

and the use of Maxwell's equations lead to (cf. Ref. 34 or Sec. 1.1 of Ref. 35)

$$
\begin{aligned}
\left.E^{\text {(out) }}(\mathbf{r})\right|_{z=D^{(+)}(x)} & =\left.E^{(\mathrm{in})}(\mathbf{r})\right|_{z=D^{(-)}(x)}, \\
{\left[\frac{\partial E^{(\text {out })}(\mathbf{r})}{\partial n}\right]_{z=D^{(+)}(x)} } & =\left[\frac{\partial E^{(\mathrm{in})}(\mathbf{r})}{\partial n}\right]_{z=D^{(-)}(x)},
\end{aligned}
$$

where $D^{(+)}$and $D^{(-)}$denote the surface profile when approached from above (vacuum) and below (dielectric), respectively, and where the normal derivative $\partial / \partial n$ is

$$
\partial / \partial n=(\hat{n} \cdot \nabla),
$$

$\hat{n}$ being the local outward normal vector

$$
\hat{n} \equiv(1 / \gamma)\{-\mathrm{d}[D(x)] / \mathrm{d} x, 1\} .
$$

$\gamma$ is defined as $\left(1+\{\mathrm{d}[D(x)] / \mathrm{d} x\}^{2}\right)^{1 / 2}$.

From the Helmholtz equations [Eqs. (6)] and those corresponding to their respective Green functions $G_{o}\left(\mathbf{r}, \mathbf{r}^{\prime}\right)$ and $G\left(\mathbf{r}, \mathbf{r}^{\prime}\right)$, we have

$$
\begin{aligned}
& G_{o}\left(\mathbf{r}, \mathbf{r}^{\prime}\right) \nabla_{\mathbf{r}^{\prime}}^{2} E^{(\text {out })}\left(\mathbf{r}^{\prime}\right)-E^{(\text {out })}\left(\mathbf{r}^{\prime}\right) \nabla_{\mathbf{r}^{\prime}}^{2} G_{o}\left(\mathbf{r}, \mathbf{r}^{\prime}\right) \\
& \quad=4 \pi \delta\left(\left|\mathbf{r}-\mathbf{r}^{\prime}\right|\right) E^{(\text {out })}\left(\mathbf{r}^{\prime}\right) \\
& \begin{aligned}
G\left(\mathbf{r}, \mathbf{r}^{\prime}\right) \nabla_{\mathbf{r}^{\prime}}^{2} E^{(\text {in) }}\left(\mathbf{r}^{\prime}\right)-E^{(\text {in) })}\left(\mathbf{r}^{\prime}\right) \nabla_{\mathbf{r}^{\prime}}{ }^{2} G\left(\mathbf{r}, \mathbf{r}^{\prime}\right) \\
=4 \pi \delta\left(\left|\mathbf{r}-\mathbf{r}^{\prime}\right|\right) E^{(\mathrm{in})}\left(\mathbf{r}^{\prime}\right)
\end{aligned}
\end{aligned}
$$

In the two-dimensional geometry associated with the onedimensional surface under consideration, the Green functions $G_{o}$ and $G$ are given by the zeroth-order Hankel function of the first kind:

$$
\begin{aligned}
G_{o}\left(\mathbf{r}, \mathbf{r}^{\prime}\right) & =\pi i H_{0}^{(1)}\left(k_{o}\left|\mathbf{r}-\mathbf{r}^{\prime}\right|\right), \\
G\left(\mathbf{r}, \mathbf{r}^{\prime}\right) & =\pi i H_{0}^{(1)}\left\{[\epsilon(\omega)]^{1 / 2} k_{o}\left|\mathbf{r}-\mathbf{r}^{\prime}\right|\right\} .
\end{aligned}
$$

Now we integrate Eqs. (10) over the two semi-infinite vol- 
umes $\tilde{V}$ and $V$ according to the following cases:

(a) Vacuum, $\mathbf{r}^{\prime} \in \tilde{V}: \quad$ By virtue of Green's theorem, the integral of Eq. (10a) may be written as

$$
\begin{aligned}
\frac{1}{4 \pi} \int_{\partial \tilde{V}} \mathrm{~d} \mathbf{r}^{\prime} \cdot\left[G_{o}\left(\mathbf{r}, \mathbf{r}^{\prime}\right) \nabla_{\mathbf{r}^{\prime}} E\left(\mathbf{r}^{\prime}\right)-E\left(\mathbf{r}^{\prime}\right) \nabla_{\mathbf{r}^{\prime}} G_{o}\left(\mathbf{r}, \mathbf{r}^{\prime}\right)\right] & =E^{\text {(out) }(\mathbf{r}),} \\
\mathbf{r} & \in \tilde{V}, \quad(13 \mathrm{a}) \\
\frac{1}{4 \pi} \int_{\partial \tilde{V}} \mathrm{~d} \mathbf{r}^{\prime} \cdot\left[G_{o}\left(\mathbf{r}, \mathbf{r}^{\prime}\right) \nabla_{\mathbf{r}^{\prime}} E\left(\mathbf{r}^{\prime}\right)-E\left(\mathbf{r}^{\prime}\right) \nabla_{\mathbf{r}^{\prime}} G_{o}\left(\mathbf{r}, \mathbf{r}^{\prime}\right)\right] & =0, \\
\mathbf{r} & \in V,
\end{aligned}
$$

where $\partial \tilde{V}$ means the limiting surface of the volume $\tilde{V}$, which can be divided into two parts: the rough surface $z=D(x)$ and a hemisphere $\Sigma^{(\infty)}$ of infinite radius in the upper half-space. As a consequence, the above integral is decomposed into the contributions

$$
\int_{\partial \tilde{V}} \mathrm{~d} \mathbf{r}^{\prime}=\int_{\Sigma^{(\infty)}} d \mathbf{r}^{\prime}+\int_{z=D(x)} d \mathbf{S}^{(-)},
$$

the random surface element $d \mathbf{S}^{(-)}$being

$$
\mathrm{d} \mathbf{S}^{(-)}=-\mathrm{d} \mathbf{S}^{(+)}=(-\hat{n}) \mathrm{d} S=-\hat{n} \gamma \mathrm{d} x^{\prime} .
$$

Taking into account that the electric vector $\mathbf{E}^{\text {(out) }}(\mathbf{r})$ in the vacuum may be written as the sum of an incident and a scattered (reflected) field, $\mathbf{E}^{(i)}(\mathbf{r})$ and $\mathbf{E}^{(r)}(\mathbf{r})$, respectively, we express its amplitude by

$$
E^{(\text {out })}(\mathbf{r})=E^{(i)}(\mathbf{r})+E^{(r)}(\mathbf{r}),
$$

and, recalling the radiation condition for the scattered field, we find that the integral over $\Sigma^{(\infty)}$ is

$$
\int_{\Sigma^{(\text {ou }}}\left[G_{0} \nabla E^{(\text {out })}-E^{\text {(out) }} \nabla G_{o}\right]=4 \pi E^{(i)} .
$$

Note that, in the case of an incident plane wave, infinity can be reached without our leaving the vicinity of the surface; thus the Sommerfeld radiation condition cannot be expressed as usual. Then, we shall say that the scattered field satisfies the radiation condition, meaning that it is outgoing in $z>D_{\max }\left\{D_{\max }=\max [D(x)]\right\}$; namely, its angular spectrum representation in $z>D_{\max }$ contains only plane-wave components propagating into $z>0$. This difficulty with this kind of geometry has been discussed in detail, for instance, in Ref. 36. A configuration in which the usual radiation condition can be directly applied, however, is obtained by the localization of the surface through illumination by an incident beam instead of an (infinitely extended) plane wave.

On introducing Eqs. (14), (15), and (17) into Eqs. (13), we obtain

$$
\begin{aligned}
E^{(i)}(\mathbf{r})+\frac{1}{4 \pi} \int_{-\infty}^{\infty} \mathrm{d} x^{\prime}\left[E^{(\text {out })}\left(\mathbf{r}^{\prime}\right) \frac{\partial G_{o}\left(\mathbf{r}, \mathbf{r}^{\prime}\right)}{\partial n^{\prime}}\right. & \\
\left.-G_{o}\left(\mathbf{r}, \mathbf{r}^{\prime}\right) \frac{\partial E^{(\text {out })}\left(\mathbf{r}^{\prime}\right)}{\partial n^{\prime}}\right] \gamma^{\prime} & =E^{(\text {out })}(\mathbf{r}), \\
\mathbf{r} & \in \tilde{V}, \quad(18 \mathrm{a})
\end{aligned}
$$

$$
\begin{aligned}
& E^{(i)}(\mathbf{r})+\frac{1}{4 \pi} \int_{-\infty}^{\infty} \mathrm{d} x^{\prime}\left[E^{\text {(out) }}\left(\mathbf{r}^{\prime}\right) \frac{\partial G_{o}\left(\mathbf{r}, \mathbf{r}^{\prime}\right)}{\partial n^{\prime}}\right. \\
&\left.-G_{o}\left(\mathbf{r}, \mathbf{r}^{\prime}\right) \frac{\partial E^{\text {(out) }}\left(\mathbf{r}^{\prime}\right)}{\partial n^{\prime}}\right] \gamma^{\prime}=0 \\
& \mathbf{r} \in V, \quad(18 \mathrm{~b})
\end{aligned}
$$

where $\mathbf{r}^{\prime}=\left[x^{\prime}, z^{\prime}=D\left(x^{\prime}\right)\right]$.

(b) Dielectric, $\mathbf{r}^{\prime} \in V$ : In contrast to what occurs in the upper half-space, no incident wave exists in the second medium. Therefore, when applying Green's theorem to the dielectric volume, we see that the integral over the lower hemisphere $\Sigma^{(-\infty)}$, equivalent to Eq. (17), vanishes by our using the radiation condition for the scattered (transmitted) field $E^{(\text {in) }}$, namely,

$$
\int_{\Sigma^{(-\infty)}}\left[G \nabla E^{(\mathrm{in})}-E^{(\mathrm{in})} \nabla G\right]=0 .
$$

With the aid of Eq. (19) and proceeding in a similar way as we did with Eqs. (18), we obtain another two equations, which now involve the field transmitted into the dielectric $\mathbf{E}^{\text {(in): }}$

$$
\begin{aligned}
-\frac{1}{4 \pi} \int_{-\infty}^{\infty} \mathrm{d} x^{\prime}\left[E^{(\mathrm{in})}\left(\mathbf{r}^{\prime}\right) \frac{\partial G\left(\mathbf{r}, \mathbf{r}^{\prime}\right)}{\partial n^{\prime}}-G\left(\mathbf{r}, \mathbf{r}^{\prime}\right) \frac{\partial E^{(\mathrm{in})}\left(\mathbf{r}^{\prime}\right)}{\partial n^{\prime}}\right] \gamma^{\prime} & =0, \\
\mathbf{r} & \in \tilde{V}, \quad(20 \mathrm{a}) \\
-\frac{1}{4 \pi} \int_{-\infty}^{\infty} \mathrm{d} x^{\prime}\left[E^{(\mathrm{in})}\left(\mathbf{r}^{\prime}\right) \frac{\partial G\left(\mathbf{r}, \mathbf{r}^{\prime}\right)}{\partial n^{\prime}}\right. & \\
\left.-G\left(\mathbf{r}, \mathbf{r}^{\prime}\right) \frac{\partial E^{(\mathrm{in})}\left(\mathbf{r}^{\prime}\right)}{\partial n^{\prime}}\right] \gamma^{\prime} & =E^{(\mathrm{in})(\mathbf{r}),} \\
\mathbf{r} & \in V . \quad(20 \mathrm{~b})
\end{aligned}
$$

The four equations [Eqs. (18) and (20)] enable us to obtain an exact solution for the scattering of an electromagnetic wave from a rough one-dimensional surface. Equation (18b) expresses how the incident field is extinguished inside the dielectric by sources generated over the surface on interaction with the medium. Equations (18a) and $(20 \mathrm{~b})$ describe how these source terms create both reflected and transmitted fields, respectively.

The aim that we pursue in this paper is to obtain the angular distribution of the field $E^{(r)}=E^{(\text {out })}-E^{(i)}$ and $E^{(t)}=E^{(\text {in) }}$ in the far zone $(|\mathbf{r}| / \lambda \gg 1)$. First, we define two unknown surface source functions:

$$
\begin{aligned}
E(x) & =E^{(\text {out })}[x, D(x)] \\
& =E^{(\text {in) }}[x, D(x)], \\
F(x) & =\gamma\left[\frac{\partial E^{(\text {out })}(\mathbf{r})}{\partial n}\right]_{z=D^{(+)}(x)} \\
& =\gamma\left[\frac{\partial E^{(\mathrm{in})}(\mathbf{r})}{\partial n}\right]_{z=D^{(-)}(x)},
\end{aligned}
$$

where the boundary values [Eqs. (7)] have been accounted for. By introducing Eqs. (21) and (22) into Eqs. (18a) and 
(20a), we have

$$
\begin{gathered}
E^{(i)}(\mathbf{r})+\frac{1}{4 \pi} \int_{-\infty}^{\infty} \mathrm{d} x^{\prime}\left\{E\left(x^{\prime}\right)\left[\frac{\partial G_{0}}{\partial z^{\prime}}-D^{\prime}\left(x^{\prime}\right) \frac{\partial G_{0}}{\partial x^{\prime}}\right]-G_{o} F\left(x^{\prime}\right)\right\} \\
=E^{(\text {out })}(\mathbf{r}), \\
-\frac{1}{4 \pi} \int_{-\infty}^{\infty} \mathrm{d} x^{\prime}\left\{E\left(x^{\prime}\right)\left[\frac{\partial G}{\partial z^{\prime}}-D^{\prime}\left(x^{\prime}\right) \frac{\partial G}{\partial x^{\prime}}\right]-G F\left(x^{\prime}\right)\right\} \\
=0 .
\end{gathered}
$$

Of course, in Eqs. (23) and (24) $\mathbf{r}$ is evaluated in the vacuum half-space $(\tilde{V})$.

By making $\mathbf{r}$ tend to a surface point $z \stackrel{\delta \rightarrow 0^{+}}{\longrightarrow} D(x)+\delta$, we have

$$
\begin{gathered}
E^{(i)}[x, D(x)]+\frac{1}{4 \pi} \int_{-\infty}^{\infty} \mathrm{d} x^{\prime}\left\{E\left(x^{\prime}\right)\left[\frac{\partial G_{o}}{\partial z^{\prime}}-D^{\prime}\left(x^{\prime}\right) \frac{\partial G_{o}}{\partial x^{\prime}}\right]\right. \\
\left.-G_{o} F\left(x^{\prime}\right)\right\}=E(x), \\
-\frac{1}{4 \pi} \int_{-\infty}^{\infty} \mathrm{d} x^{\prime}\left\{E\left(x^{\prime}\right)\left[\frac{\partial G}{\partial z^{\prime}}-D^{\prime}\left(x^{\prime}\right) \frac{\partial G}{\partial x^{\prime}}\right]-G F\left(x^{\prime}\right)\right\}=0,
\end{gathered}
$$

where $G_{o}$ and $G$ must be used according to expressions (11) and (12): Much care for the singularities of the Hankel functions $H_{0}^{(1)}$ and $H_{1}^{(1)}$ has to be taken when the numerical computation of Eqs. (25) is done. Whereas the singularity of $H_{0}^{(1)}$ for $\mathbf{r}=\mathbf{r}^{\prime}$ is integrable, that of $H_{1}^{(1)}$ is not. These singularities are extracted following App. A of Ref. 6.

Once $E$ and $F$ are calculated from the system of coupled integral equations [Eqs. (25)] with the singularities at $\mathbf{r}=\mathbf{r}^{\prime}$ extracted, we can obtain $E^{(r)}$ and $E^{(t)}$ by introducing $E$ and $F$ into Eqs. (18a) and (20b). Since we are interested in the far-zone intensity, we take the asymptotic expressions for the Hankel functions ${ }^{37}$ as $k\left|\mathbf{r}_{>,<}-\mathbf{r}^{\prime}\right| \rightarrow \infty$ in a fixed direction. Namely, we make the expansion

$$
\left|\mathbf{r}_{>,<}-\mathbf{r}^{\prime}\right| \approx \mathbf{r}_{>,<}-\left|\mathbf{r}_{>,<} \cdot \mathbf{r}^{\prime}\right|,
$$

$r_{>}$and $r_{<}$representing the moduli of the position vectors in the far vacuum and dielectric zone, respectively. Accordingly, the scattered field above the surface (reflected) and below the surface (transmitted) may be written in the form

$$
\begin{aligned}
E^{(r)}\left(r_{>}, \theta\right)= & \frac{\exp \left[i\left(k_{o} r_{>}-\pi / 4\right)\right]}{2\left(2 \pi k_{o} r_{>}\right)^{1 / 2}} \\
& \times \int_{-\infty}^{\infty} \mathrm{d} x^{\prime}\left\{k_{o}\left[\cos \theta-D^{\prime}\left(x^{\prime}\right) \sin \theta\right] E\left(x^{\prime}\right)\right. \\
& \left.-i F\left(x^{\prime}\right)\right\} \exp \left(-i \mathbf{K} \cdot \boldsymbol{r}^{\prime}\right), \quad(2 \\
E^{(t)}\left(r_{>}, \theta_{t}\right)= & \frac{\exp \left[i\left(\sqrt{\epsilon} k_{o} r_{<}-\pi / 4\right)\right]}{2\left(2 \pi \sqrt{\epsilon} k_{o} r_{<}\right)^{1 / 2}} \\
& \times \int_{-\infty}^{\infty} \mathrm{d} x^{\prime}\left\{\sqrt{\epsilon} k_{o}\left[\cos \theta_{t}+D^{\prime}\left(x^{\prime}\right) \sin \theta_{t}\right] E\left(x^{\prime}\right)\right. \\
& \left.\quad+i F\left(x^{\prime}\right)\right\} \exp \left(-i \mathbf{K}_{t} \cdot \boldsymbol{r}^{\prime}\right),
\end{aligned}
$$

$\theta$ and $\theta_{t}$ being the angles of observation above the surface and below the surface, respectively (see Fig. 1). Note that so far no restriction has been imposed on the dielectric constant. For Eq. (26b) to be valid, the transmitted field should be propagating; i.e., $\Re(\epsilon)>0$. In any other case, the transmitted field would be evanescent, and then Eq. (26b) would be zero. On the other hand, Eq. (26a) remains valid whatever the value of $\epsilon(\omega)$ is. Besides, no assumption is made about the surface apart from its one dimensionality; concerning this point, Eqs. (25) and (26) are completely general. From now on, we will develop a formalism specific for random surfaces, which can be extended to deterministic surfaces by suppressing the statistical averages.

In practice, a finite length $L$ of the surface is illuminated; therefore the $x$ integral in Eqs. (25) and (26) is extended to the $L$ interval only. Let us denote by $I_{o}$ the total power flow, or integrated intensity, of the incident wave:

$$
I_{o} \propto\left|E^{(i)}\right|^{2} L \cos \theta_{o} .
$$

Then the mean scattered intensities (reflected and transmitted, respectively), normalized to the incident power flow, will be

$$
\begin{aligned}
\left(1 / I_{o}\right)\left\langle I_{s}^{(r)}(\theta)\right\rangle & =\left(r_{>} / I_{o}\right)\left\langle\left|E^{(r)}\left(r_{>}, \theta\right)\right|^{2}\right\rangle, \\
\left(1 / I_{o}\right)\left\langle I_{s}^{(t)}\left(\theta_{t}\right)\right\rangle & =\sqrt{\epsilon}\left(r_{<} / I_{o}\right)\left\langle\left|E^{(t)}\left(r_{<}, \theta_{t}\right)\right|^{2}\right\rangle,
\end{aligned}
$$

with $\langle\cdot\rangle$ denoting the average over the ensemble of realizations of $D(x)$. The $\sqrt{\epsilon}$ factor that appears on the righthand side of Eq. (28b) is due to the dependence of the Poynting vector on the electric field:

$$
(4 \pi / c)|\mathbf{S}|=(\epsilon / \mu)^{1 / 2}|\mathbf{E}|^{2}=(\mu / \epsilon)^{1 / 2}|\mathbf{H}|^{2} .
$$

Finally, by introducing expressions (26) and (27) into Eqs. (28), we obtain the normalized mean scattered intensities in the far zone for $s$ polarization. The mean incoherent scattered intensities are easily calculated from

$$
\left(1 / I_{o}\right)\left\langle\Delta I_{s}^{(r)}(\theta)\right\rangle=\left(r_{>} / I_{o}\right)\left[\left\langle\left|E^{(r)}\left(r_{>}, \theta\right)\right|^{2}\right\rangle-\left|\left\langle E^{(r)}\left(r_{>}, \theta\right)\right\rangle\right|^{2}\right],
$$

$\left(1 / I_{o}\right)\left\langle\Delta I_{s}^{(t)}\left(\theta_{t}\right)\right\rangle=\sqrt{\epsilon}\left(r_{<} / I_{o}\right)\left[\left\langle\left|E^{(t)}\left(r_{<}, \theta_{t}\right)\right|^{2}\right\rangle-\left|\left\langle E^{(t)}\left(r_{<}, \theta_{t}\right)\right\rangle\right|^{2}\right]$.

On integrating the mean scattering intensities [Eqs. (28)] over every scattering angle, we derive an expression both for the total normalized reflected intensity (reflectance $R$ )

$$
R=\frac{1}{I_{o}} \int_{-\pi / 2}^{\pi / 2}\left\langle I^{(r)}(\theta)\right\rangle \mathrm{d} \theta
$$

and for the total normalized transmitted intensity (transmittance $T$ )

$$
T=\frac{1}{I_{o}} \int_{-\pi / 2}^{\pi / 2}\left\langle I^{(t)}\left(\theta_{t}\right)\right\rangle \mathrm{d} \theta_{t}
$$

In the absence of absorption inside the dielectric me$\operatorname{dium}[\Re(\epsilon)>0, \mathfrak{I}(\epsilon)=0]$, the unitarity condition must be satisfied:

$$
R+T=1
$$

\section{2. $p$ Polarization}

In the case of $p$ polarization, the Helmholtz equations for the complex amplitude of the magnetic vector, which now 
has a $y$ component only, are

$$
\begin{aligned}
& \nabla^{2} H^{\text {(out) }}\left(\mathbf{r}^{\prime}\right)+k_{o}{ }^{2} H^{\text {(out) }}(\mathbf{r})=0, \quad z>D(x),(\mathbf{r} \in \tilde{V}), \\
& \nabla^{2} H^{(\mathrm{in})}\left(\mathbf{r}^{\prime}\right)+\epsilon(\omega) k_{0}^{2} H^{(\mathrm{in})}(\mathbf{r})=0, \quad z<D(x),(\mathbf{r} \in V),
\end{aligned}
$$

the superscripts (out) and (in) having the same meaning as in Subsection 2.A.1. Also, the amplitude of the magnetic field and its derivative satisfy the following boundary conditions across the surface:

$$
\begin{aligned}
\left.H^{(\mathrm{out})}(\mathbf{r})\right|_{z=D^{(+)}(x)} & =\left.H^{(\mathrm{in})}(\mathbf{r})\right|_{z=D^{(-)}(x)}, \\
{\left[\frac{\partial H^{(\mathrm{out})}(\mathbf{r})}{\partial n}\right]_{z=D^{(+)}(x)} } & =\frac{1}{\epsilon(\omega)}\left[\frac{\partial H^{(\mathrm{in})}(\mathbf{r})}{\partial n}\right]_{z=D^{(-)}(x)} .
\end{aligned}
$$

Operating as in to the case of $s$ polarization, we finally arrive at the four equations that form the basis for our obtaining the solution of the scattering problem: and $H_{1}^{(1)}$ extracted as mentioned in Subsection 2.A.1]:

$$
\begin{aligned}
& H^{(i)}[x, D(x)]+\frac{1}{4 \pi} \int_{-\infty}^{\infty} \mathrm{d} x^{\prime} \\
& \times\left\{H\left(x^{\prime}\right)\left[\frac{\partial G_{0}}{\partial z^{\prime}}-D^{\prime}\left(x^{\prime}\right) \frac{\partial G_{o}}{\partial x^{\prime}}\right]-G_{o} L\left(x^{\prime}\right)\right\}=H(x), \quad(41 \mathrm{a}) \\
& -\frac{1}{4 \pi} \int_{-\infty}^{\infty} \mathrm{d} x^{\prime}\left\{H\left(x^{\prime}\right)\left[\frac{\partial G}{\partial z^{\prime}}-D^{\prime}\left(x^{\prime}\right) \frac{\partial G}{\partial x^{\prime}}\right]-\epsilon(\omega) G L\left(x^{\prime}\right)\right\}=0 .
\end{aligned}
$$

Owing to the dependence of the Poynting vector on the magnetic field [Eq. (29)], the mean scattered intensities are now

$$
\begin{aligned}
\left(1 / I_{o}\right)\left\langle I_{p}^{(r)}(\theta)\right\rangle & =\left(r_{>} / I_{o}\right)\left\langle\left|H^{(r)}\left(r_{>}, \theta\right)\right|^{2}\right\rangle, \\
\left(1 / I_{o}\right)\left\langle I_{p}^{(t)}\left(\theta_{t}\right)\right\rangle & =(1 / \sqrt{\epsilon})\left(r_{<} / I_{o}\right)\left\langle\left|H^{(t)}\left(r_{<}, \theta_{t}\right)\right|^{2}\right\rangle,
\end{aligned}
$$

which we straightforwardly calculate by taking into account the far-zone expressions for the complex amplitudes

$$
\begin{aligned}
& H^{(i)}(\mathbf{r})+\frac{1}{4 \pi} \int_{-\infty}^{\infty} \mathrm{d} x^{\prime}\left[H^{\text {(out) }}\left(\mathbf{r}^{\prime}\right) \frac{\partial G_{o}\left(\mathbf{r}, \mathbf{r}^{\prime}\right)}{\partial n^{\prime}}-G_{o}\left(\mathbf{r}, \mathbf{r}^{\prime}\right) \frac{\partial H^{(\text {out })}\left(\mathbf{r}^{\prime}\right)}{\partial n^{\prime}}\right] \gamma^{\prime}=H^{\text {(out) }}(\mathbf{r}), \quad \mathbf{r} \in \tilde{V}, \\
& H^{(i)}(\mathbf{r})+\frac{1}{4 \pi} \int_{-\infty}^{\infty} \mathrm{d} x^{\prime}\left[H^{\text {(out) }}\left(\mathbf{r}^{\prime}\right) \frac{\partial G_{o}\left(\mathbf{r}, \mathbf{r}^{\prime}\right)}{\partial n^{\prime}}-G_{o}\left(\mathbf{r}, \mathbf{r}^{\prime}\right) \frac{\partial H^{(\text {out })}\left(\mathbf{r}^{\prime}\right)}{\partial n^{\prime}}\right] \gamma^{\prime}=0, \quad \mathbf{r} \in V, \\
& -\frac{1}{4 \pi} \int_{-\infty}^{\infty} \mathrm{d} x^{\prime}\left[H^{(\mathrm{in})}\left(\mathbf{r}^{\prime}\right) \frac{\partial G\left(\mathbf{r}, \mathbf{r}^{\prime}\right)}{\partial n^{\prime}}-G\left(\mathbf{r}, \mathbf{r}^{\prime}\right) \frac{\partial H^{(\mathrm{in})}\left(\mathbf{r}^{\prime}\right)}{\partial n^{\prime}}\right] \gamma^{\prime}=0, \quad \mathbf{r} \in \tilde{V}, \\
& -\frac{1}{4 \pi} \int_{-\infty}^{\infty} \mathrm{d} x^{\prime}\left[H^{(\mathrm{in})}\left(\mathbf{r}^{\prime}\right) \frac{\partial G\left(\mathbf{r}, \mathbf{r}^{\prime}\right)}{\partial n^{\prime}}-G\left(\mathbf{r}, \mathbf{r}^{\prime}\right) \frac{\partial H^{(\mathrm{in})}\left(\mathbf{r}^{\prime}\right)}{\partial n^{\prime}}\right] \gamma^{\prime}=H^{(\mathrm{in})}(\mathbf{r}), \quad \mathbf{r} \in V
\end{aligned}
$$

The source functions $H$ and $L$ are now defined as

$$
\begin{aligned}
H(x) & =H^{(\text {out })}[x, D(x)] \\
& =H^{(\text {in) }}[x, D(x)], \\
L(x) & =\gamma \frac{\partial H^{\text {(out) }}(\mathbf{r})}{\partial n} \\
& =\frac{\gamma}{\epsilon(\omega)} \frac{\partial H^{(\text {in })}(\mathbf{r})}{\partial n} .
\end{aligned}
$$

From Eqs. (36a), (37b), (38), and (39) the far fields are

$$
\begin{aligned}
H^{(r)}\left(r_{>}, \theta\right)= & \frac{\exp \left[i\left(k_{o} r_{>}-\pi / 4\right)\right]}{2\left(2 \pi k_{o} r_{>}\right)^{1 / 2}} \\
& \times \int_{-\infty}^{\infty} \mathrm{d} x^{\prime}\left\{k_{o}\left[\cos \theta-D^{\prime}\left(x^{\prime}\right) \sin \theta\right] H\left(x^{\prime}\right)\right. \\
& \left.-i L\left(x^{\prime}\right)\right\} \exp \left(-i \mathbf{K} \cdot \boldsymbol{r}^{\prime}\right), \quad(40) \\
H^{(t)}\left(r_{>}, \theta_{t}\right)= & \frac{\exp \left[i\left(\sqrt{\epsilon} k_{o} r_{<}-\pi / 4\right)\right]}{2\left(2 \pi \sqrt{\epsilon} k_{o} r_{<}\right)^{1 / 2}} \\
& \times \int_{-\infty}^{\infty} \mathrm{d} x^{\prime}\left\{\sqrt{\epsilon} k_{o}\left[\cos \theta_{t}+D^{\prime}\left(x^{\prime}\right) \sin \theta_{t}\right] H\left(x^{\prime}\right)\right. \\
& \left.\quad+i \epsilon L\left(x^{\prime}\right)\right\} \exp \left(-i \mathbf{K}_{t} \cdot \boldsymbol{r}^{\prime}\right), \quad
\end{aligned}
$$

with $H$ and $L$ being the solutions of the following coupled integral equations [of course, with the singularities of $H_{0}^{(1)}$ of the magnetic fields [Eqs. (40)]. The reflectance and the transmittance are obtained in the same manner as in Eqs. (31) and (32). Hence the unitarity condition [Eq. (33)] remains valid under the restriction mentioned above, namely, a lossless dielectric medium.

\section{B. Kirchhoff Approximation}

For the KA, also known as the physical-optics and the tangent-plane methods, it is assumed that the surface can be replaced at each point by its tangent plane. ${ }^{21-23}$ This means that the field on the surface can be considered the addition of the incident field and the reflected field, with the use of the Fresnel coefficients. Thus, within the scope of this approach, the field and its derivative on the surface (from the vacuum side) are written as follows (cf. p. 20 of Ref. 21):

for $s$ polarization,

$$
\begin{aligned}
E^{\text {(out) }}[x, D(x)] & =\left[1+R_{s}(x)\right] E^{(i)}[x, D(x)], \\
{\left[\frac{\partial E^{(\text {out })}(\mathbf{r})}{\partial n}\right]_{z=D^{(+)}(x)} } & =i \mathbf{K}_{o} \cdot \hat{n}\left[1-R_{s}(x)\right] E^{(i)}[x, D(x)],
\end{aligned}
$$

for $p$ polarization,

$$
\begin{aligned}
H^{\text {(out) }[x, D(x)]} & =\left[1+R_{p}(x)\right] H^{(i)}[x, D(x)], \\
{\left[\frac{\partial H^{(\text {out })}(\mathbf{r})}{\partial n}\right]_{z=D^{(+)}(x)} } & =i \mathbf{K}_{o} \cdot \hat{n}\left[1-R_{p}(x)\right] H^{(i)}[x, D(x)],
\end{aligned}
$$




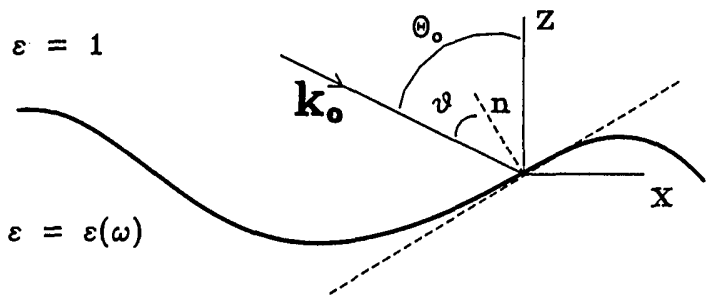

Fig. 2. Illustration of the local angle of incidence $\vartheta(x)$ at the tangent plane.

$\hat{n}$ being the local outward normal deviated from the $\hat{z}$ direction an angle $\alpha=\arctan \left[D^{\prime}(x)\right]$ and $R_{s}(x), R_{p}(x)$ being the local Fresnel coefficients ${ }^{35}$

$$
\begin{aligned}
& R_{s}(x)=\frac{\cos \vartheta(x)-\sqrt{\epsilon} \cos \vartheta_{t}(x)}{\cos \vartheta(x)+\sqrt{\epsilon} \cos \vartheta_{t}(x)} \\
& R_{p}(x)=\frac{\sqrt{\epsilon} \cos \vartheta(x)-\cos \vartheta_{t}(x)}{\sqrt{\epsilon} \cos \vartheta(x)+\cos \vartheta_{t}(x)}
\end{aligned}
$$

$\vartheta(x)$ is the angle between $\mathbf{K}_{o}$ and $\hat{n}$ (see Fig. 2), namely,

$$
\vartheta(x)=\theta_{o}-\alpha=\theta_{o}-\arctan D^{\prime}(x),
$$

and $\vartheta_{t}(x)$ denotes the local refracted angle

$$
\sin \vartheta_{t}(x)=\frac{\sin \vartheta(x)}{\sqrt{\epsilon}}
$$

Therefore the far-zone fields are straightforwardly obtained from Eqs. (26) and (40) with the substitution for the source terms $E$ and $F$ ( $H$ and $L$ ) [Eqs. (21), (22), (38), and (39)] of their expressions given by the $\mathrm{KA}$, i.e., with Eqs. (43) and (44), namely, of

$$
\begin{aligned}
E^{\mathrm{KA}}[x, D(x)]= & {\left[1+R_{s}(x)\right] E^{(i)} } \\
& \times \exp \left\{i k_{o}\left[x \sin \theta_{o}-D(x) \cos \theta_{o}\right]\right\},
\end{aligned}
$$

of the incident light. However, we shall see in Subsection 3.B that, as a result of the lower reflectivity of a dielectric interface, the range of validity of this approximation is broader for dielectric media than that accepted for perfect conductors.

\section{Numerical Implementation}

To date, the most accurate way to solve the scattering equations is to treat them numerically. Thus, by means of a quadrature scheme, the integration is converted into a summation, once the infinite limits of surface integration are replaced by the finite length $(-L / 2, L / 2)$. Proceeding according to the numerical method described in Refs. 5-8, we sample each surface profile with $N$ points and then convert the systems of integral equations [Eqs. (25) and (41)] into two systems of linear equations as follows:

for $s$ polarization,

$$
\left[\begin{array}{cc}
\mathbf{A}^{(\mathbf{o})}+\mathbf{I} & \mathbf{B}^{(\mathbf{o})} \\
\mathbf{A}-\mathbf{I} & \mathbf{B}
\end{array}\right]\left[\begin{array}{l}
\mathbf{E} \\
\mathbf{F}
\end{array}\right]=2\left[\begin{array}{c}
\mathbf{E}^{(\mathbf{i})} \\
\mathbf{0}
\end{array}\right]
$$

for $p$ polarization,

$$
\left[\begin{array}{cc}
\mathbf{A}^{(\mathbf{o})}+\mathbf{I} & \mathbf{B}^{(\mathbf{o})} \\
\mathbf{A}-\mathbf{I} & \epsilon(\omega) \mathbf{B}
\end{array}\right]\left[\begin{array}{l}
\mathbf{H} \\
\mathbf{L}
\end{array}\right]=2\left[\begin{array}{c}
\mathbf{H}^{(\mathbf{i})} \\
\mathbf{0}
\end{array}\right]
$$

The vectors $\mathbf{E}^{(\mathbf{i})}, \mathbf{H}^{(\mathbf{i})}, \mathbf{E}, \mathbf{F}, \mathbf{H}$, and $\mathbf{L}$ have components $E_{n}^{(i)}, H_{n}^{(i)}, E_{n}, F_{n}, H_{n}$, and $L_{n}$, respectively, which are the functions $E^{(i)}(x), H^{(i)}(x), E(x), F(x), H(x)$, and $L(x)$ evaluated at each sampling point $x_{n}$ of the surface, viz.,

$$
\begin{aligned}
E_{n}^{(i)} & =E^{(i)}\left(x_{n}\right), & H_{n}^{(i)} & =H^{(i)}\left(x_{n}\right), \\
E_{n} & =E\left(x_{n}\right), & F_{n} & =F\left(x_{n}\right), \\
H_{n} & =H\left(x_{n}\right), & L_{n} & =L\left(x_{n}\right),
\end{aligned}
$$

where $x_{n}=-L / 2+(n-1 / 2) \Delta x(\Delta x=L / N, n=1, \ldots$, $N)$. The matrices $\mathbf{A}$ and $\mathbf{B}$ have elements that are

$$
\begin{aligned}
A_{m n} & =\left\{\begin{array}{lr}
\frac{i \sqrt{\epsilon} k_{o} \Delta x}{2} \frac{D^{\prime}\left(x_{n}\right)\left(x_{m}-x_{n}\right)-\left[D\left(x_{m}\right)-D\left(x_{n}\right)\right]}{\left\{\left(x_{m}-x_{n}\right)^{2}+\left[D\left(x_{m}\right)-D\left(x_{n}\right)\right]^{2}\right\}^{1 / 2}} H_{1}^{(1)}\left(\sqrt{\epsilon} k_{o}\left\{\left(x_{m}-x_{n}\right)^{2}+\left[D\left(x_{m}\right)-D\left(x_{n}\right)\right]^{2}\right\}^{1 / 2}\right), & m \neq n \\
-\frac{\Delta x}{2 \pi \gamma^{-2}} D^{\prime \prime}\left(x_{n}\right), & m=n
\end{array},\right. \\
B_{m n} & =\left\{\begin{array}{ll}
(i \Delta x / 2) H_{0}^{(1)}\left(\sqrt{\epsilon} k_{o}\left\{\left(x_{m}-x_{n}\right)^{2}+\left[D\left(x_{m}\right)-D\left(x_{n}\right)\right]^{2}\right\}^{1 / 2}\right), & m \neq n \\
(i \Delta x / 2) H_{0}^{(1)}\left(\sqrt{\epsilon} k_{0} \gamma \Delta x / 2 e\right), & m=n
\end{array} .\right.
\end{aligned}
$$

$$
\begin{aligned}
F^{\mathrm{KA}}[x, D(x)]= & i \gamma \mathbf{K}_{o} \cdot \hat{n}\left[1-R_{s}(x)\right] E^{(i)} \\
& \times \exp \left\{i k_{o}\left[x \sin \theta_{o}-D(x) \cos \theta_{o}\right]\right\}, \\
H^{\mathrm{KA}}[x, D(x)]= & {\left[1+R_{p}(x)\right] H^{(i)} } \\
& \times \exp \left\{i k_{o}\left[x \sin \theta_{o}-D(x) \cos \theta_{o}\right]\right\}, \\
L^{\mathrm{KA}}[x, D(x)]= & i \gamma \mathbf{K}_{o} \cdot \hat{n}\left[1-R_{p}(x)\right] H^{(i)} \\
& \times \exp \left\{i k_{o}\left[x \sin \theta_{o}-D(x) \cos \theta_{o}\right]\right\} .
\end{aligned}
$$

From these far fields, calculated within the KA, Eqs. (28) and (42) yield the corresponding mean scattered (reflected and transmitted) intensities for $s$ and $p$ polarization, respectively.

In conductors, the $\mathrm{KA}$ is constrained to surfaces whose radii of curvature are much larger than the wavelength $\lambda$
I is the unit matrix whose elements are

$$
\delta_{m n}=\left\{\begin{array}{ll}
0, & m \neq n \\
1, & m=n
\end{array} .\right.
$$

The elements $A_{m n}^{(o)}$ and $B_{m n}^{(o)}$ of $\mathbf{A}^{(\mathbf{o})}$ and $\mathbf{B}^{(\mathbf{o})}$ are also defined from expressions (55) but with the use of the vacuum dielectric constant $(\epsilon=1)$ instead of $\epsilon$. Observe that the aforementioned singularities at vanishing arguments of the Hankel functions, which occur in the main diagonal of the matrix, have been rigorously integrated. Although the singularity of $H_{1}^{(1)}$ is not integrable in principle, it actually becomes so if we consider the factor that multiplies it. By replacing the integration by a summation in Eqs. (26) and (40), we obtain, for $s$ polarization [Eqs. (28)], 


$$
\begin{aligned}
& \frac{1}{I_{o}}\left\langle I_{s}^{(r)}(\theta)\right\rangle= \frac{1}{8 \pi k_{o} L \cos \theta_{o}} \\
& \times\langle| \Delta x \sum_{n=1}^{N}\left\{k_{o}\left[\cos \theta-D^{\prime}\left(x_{n}\right) \sin \theta\right] E_{n}-i F_{n}\right\} \\
&\left.\times\left.\exp \left\{-i k_{o}\left[x_{n} \sin \theta+D\left(x_{n}\right) \cos \theta\right]\right\}\right|^{2}\right\rangle, \\
& \frac{1}{I_{o}}\left\langle I_{s}^{(t)}\left(\theta_{t}\right)\right\rangle= \frac{1}{8 \pi k_{o} L \cos \theta_{o}} \\
& \times\langle| \Delta x \sum_{n=1}^{N}\left\{\sqrt{\epsilon} k_{o}\left[\cos \theta_{t}+D^{\prime}\left(x_{n}\right) \sin \theta_{t}\right] E_{n}+i F_{n}\right\} \\
&\left.\times\left.\exp \left\{-i \sqrt{\epsilon} k_{o}\left[x_{n} \sin \theta_{t}-D\left(x_{n}\right) \cos \theta_{t}\right]\right\}\right|^{2}\right\rangle,
\end{aligned}
$$

and, for $p$ polarization [Eqs. (42)]

$$
\begin{aligned}
& \frac{1}{I_{o}}\left\langle I_{p}^{(r)}(\theta)\right\rangle= \frac{1}{8 \pi k_{o} L \cos \theta_{o}} \\
& \times\langle| \Delta x \sum_{n=1}^{N}\left\{k_{o}\left[\cos \theta-D^{\prime}\left(x_{n}\right) \sin \theta\right] H_{n}-i L_{n}\right\} \\
&\left.\times\left.\exp \left\{-i k_{o}\left[x_{n} \sin \theta+D\left(x_{n}\right) \cos \theta\right]\right\}\right|^{2}\right\rangle, \\
& \frac{1}{I_{o}}\left\langle I_{p}^{(t)}\left(\theta_{t}\right)\right\rangle= \frac{\epsilon^{-1}}{8 \pi k_{o} L \cos \theta_{o}} \\
& \times\langle| \Delta x \sum_{n=1}^{N}\left\{\sqrt{\epsilon} k_{o}\left[\cos \theta_{t}+D^{\prime}\left(x_{n}\right) \sin \theta_{t}\right] H_{n}+i \epsilon L_{n}\right\} \\
&\left.\quad \times\left.\exp \left\{-i \sqrt{\epsilon} k_{o}\left[x_{n} \sin \theta_{t}-D\left(x_{n}\right) \cos \theta_{t}\right]\right\}\right|^{2}\right\rangle,
\end{aligned}
$$$$
-P \text { waves } N_{p}=400
$$

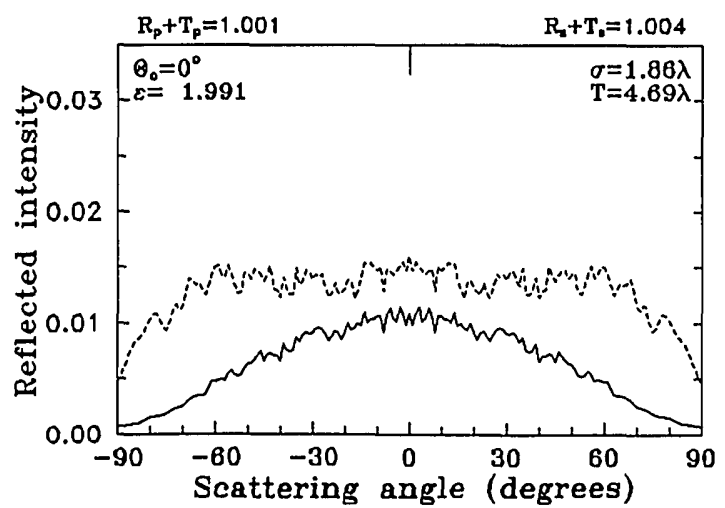

And finally, by introducing $E_{n}$ and $F_{n}$ and $H_{n}$ and $L_{n}$ [solutions of Eqs. (52) and (53)] into Eqs. (57) and (58), we find the mean scattered (reflected and transmitted) intensities for $s$ and $p$ polarization.

The above equations for the angular distribution of the mean scattered intensity give the KA solution if we change the exact boundary values $E$ and $F$ ( $H$ and $L$ ) to those obtained from Eqs. (48)-(51), whose discretized values are now written in the following form:

$$
\begin{aligned}
& E_{n}{ }^{\mathrm{KA}}= {\left[1+R_{s}\left(x_{n}\right)\right] \exp \left\{i k_{o}\left[x_{n} \sin \theta_{o}-D\left(x_{n}\right) \cos \theta_{o}\right]\right\}, } \\
& F_{n}^{\mathrm{KA}}=-i k_{o}\left[D^{\prime}\left(x_{n}\right) \sin \theta_{o}+\cos \theta_{o}\right]\left[1-R_{s}\left(x_{n}\right)\right] \\
& \quad \times \exp \left\{i k_{o}\left[x_{n} \sin \theta_{o}-D\left(x_{n}\right) \cos \theta_{o}\right]\right\}, \\
& H_{n}^{\mathrm{KA}}=\left[1+R_{p}\left(x_{n}\right)\right] \exp \left\{i k_{o}\left[x_{n} \sin \theta_{o}-D\left(x_{n}\right) \cos \theta_{o}\right]\right\}, \\
& L_{n}{ }^{\mathrm{KA}}=-i k_{o}\left[D^{\prime}\left(x_{n}\right) \sin \theta_{o}+\cos \theta_{o}\right]\left[1-R_{p}\left(x_{n}\right)\right] \\
& \quad \times \exp \left\{i k_{o}\left[x_{n} \sin \theta_{o}-D\left(x_{n}\right) \cos \theta_{o}\right]\right\},
\end{aligned}
$$

Notice that, since the final results are rigorously normalized, the complex amplitudes $E^{(i)}$ and $H^{(i)}$ are omitted in all the numerical expressions.

\section{Random Rough Surface Model}

The surface profile function $z=D(x)$ is assumed to be a statistically homogeneous and isotropic random process

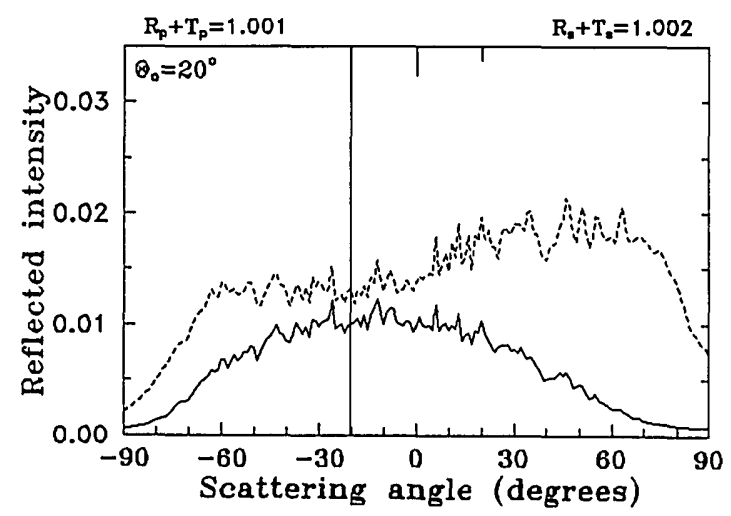

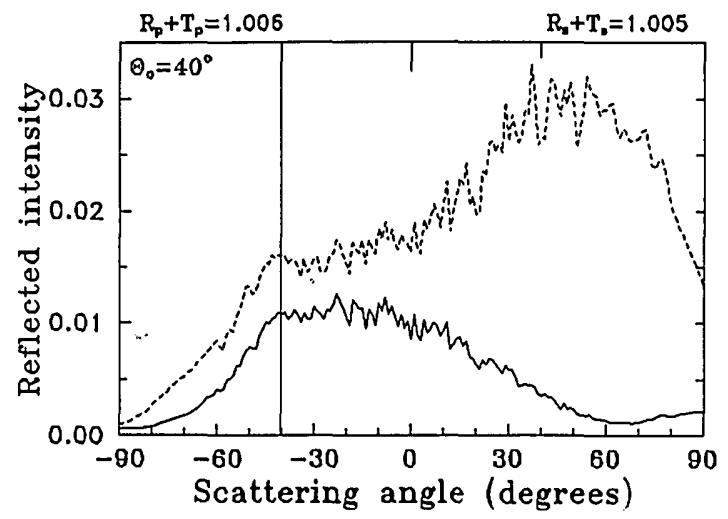

Fig. 3. Angular distribution of mean reflected intensity from a dielectric surface with $\sigma=1.86 \lambda, T=4.69 \lambda$, and $\epsilon=1.991$, at $\theta_{o}=0^{\circ}$ $20^{\circ}$, and $40^{\circ}$ (dashed curves, $s$ polarization; solid curves, $p$ polarization). The average is over 400 samples. The specular direction is shown by the mark at the upper right. The backscattering direction is marked by vertical lines. The unitarity is as shown. 


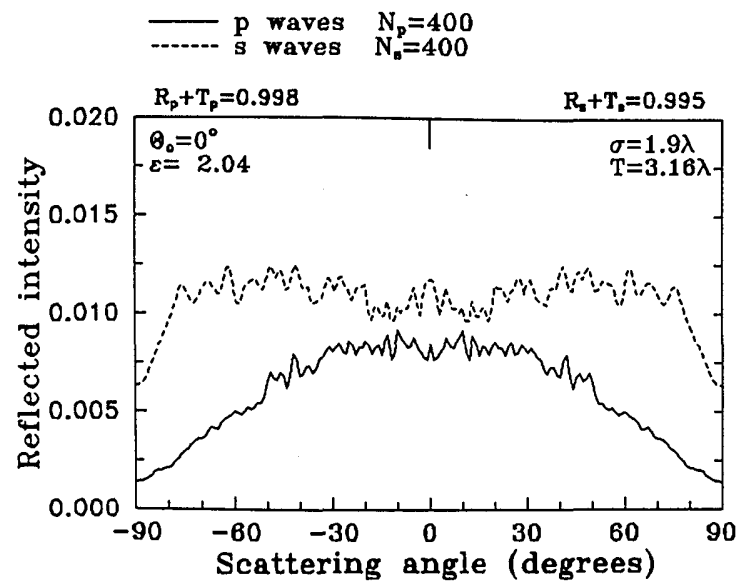

- p waves $\mathrm{N}_{\mathrm{p}}=360$

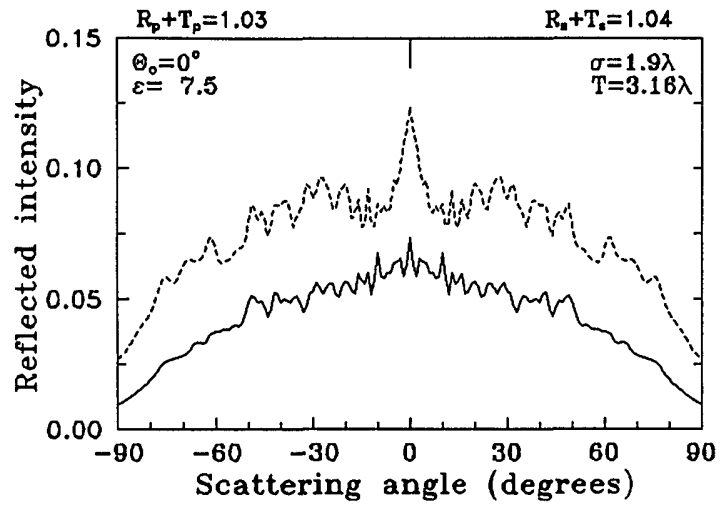

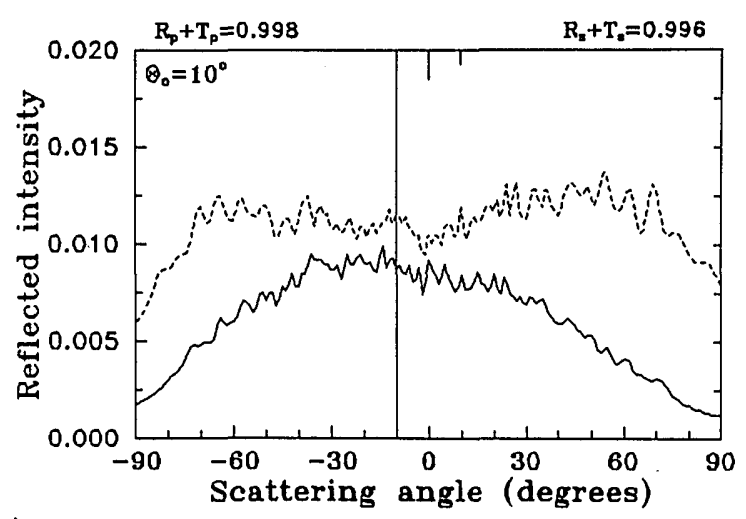

(a)

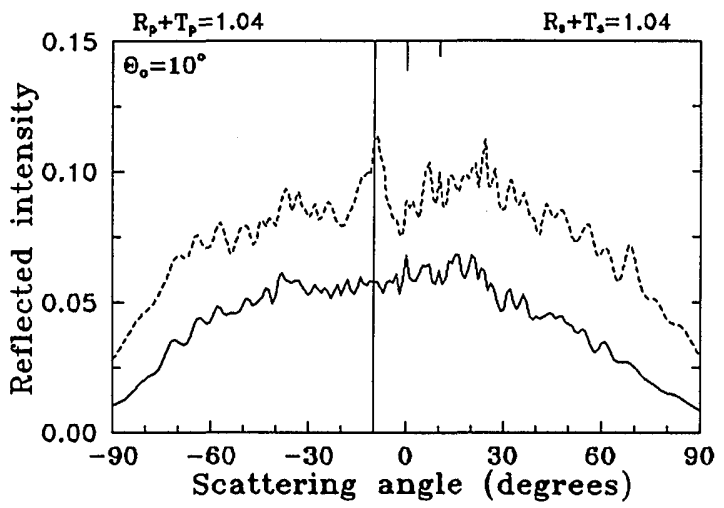

(b)

Fig. 4. Same as Fig. 3 for $\sigma=1.9 \lambda$ and $T=3.16 \lambda ; \theta_{o}=0^{\circ}$, and $10^{\circ}$ : (a) $\epsilon=2.04$, (b) $\epsilon=7.5$. described by the following statistical properties:

(1) A mean deviation from $z=0$; that is,

$$
\langle D(x)\rangle=0 .
$$

(2) Normal statistics with rms deviation $\sigma$ given by

$$
\sigma=[\langle D(x) D(x)\rangle]^{1 / 2} .
$$

(3) A Gaussian correlation function $c(\tau)$ whose width defines a correlation length $T$ as

$$
c(\tau)=\left(1 / \sigma^{2}\right)\langle D(x) D(x+\tau)\rangle=\exp \left[-\left(\tau^{2} / T^{2}\right)\right] .
$$

The surface profiles are generated by the Monte Carlo method used in Ref. 33 and further developed in Refs. 5-8. This procedure transforms a sequence of random numbers, uniformly distributed between $(0,1)$ and directly generated by the computer into a sequence (typically of $10^{5}$ numbers) with normal statistics, zero mean, and unity variance. The appropriate surface-profile sequence with Gaussian correlation function is obtained after the former sequence is correlated with a Gaussian function.

For each sample of length $L$ of the surface profile, the scattered intensities are calculated by considering plane waves incident at angles $\theta_{o},-\theta_{o}$ (where $\theta_{o}$ takes on a few values, typically $0^{\circ}, 10^{\circ}, 20^{\circ}, 30^{\circ}, 40^{\circ}, 50^{\circ}, 60^{\circ}$, and $70^{\circ}$ ). The angular distribution of intensity calculated for $-\theta_{0}$ can be regarded as the mirror image of that resulting from a plane wave incident at $\theta_{o}$ upon the surface. Hence, in this way, we double the effective number of samples over which the average is made. For normal incidence, this procedure is equivalent to that of the symmetrization of the resulting mean distribution. Nevertheless, since the asymmetry proves to be almost insignificant, this symmetrization does not significantly alter the accuracy of the average. Calculations were carried out on a CDC Cyber 180/855. For the sake of both speed and memory, 220 and 250 sampling points have been chosen for each sample of length $L$ for the ET method and the KA, respectively. The number of effective samples $\left(2 N_{s}\right)$ over which the average is made varies between 200 and 400, depending on the roughness regime studied. Typical values assigned to the length $L$ of each sample are between $20 \lambda$ and $40 \lambda$, depending on $T$.

\section{Numerical Results}

\section{A. Results from the Extinction Theorem}

In Section 2 we explained how to solve the ET equations numerically [see Eqs. (52)-(58)] when an $s$ - or $p$-polarized plane wave is incident upon a randomly rough dielectric surface. The mean scattered (reflected and transmitted) intensity so obtained is plotted for different values of the surface statistical parameters ( $\sigma$ and $T$ ); the mean incoherently scattered intensity is calculated too [Eqs. (30)]. Curves displaying the reflectance [Eq. (31)] versus the angle of incidence are also given. The unitarity condition 
[Eq. (33)] is satisfied within an error smaller than $2 \%$, except for very large angles of incidence (generally, $\theta_{0}$ greater than $70^{\circ}$, or $50^{\circ}$ for very high $\epsilon$ and $\sigma$ ). Two main cases are studied concerning the relationship between the correlation length $T$ and the wavelength of the light $\lambda$.

\section{Correlation Length Larger Than the Wavelength $(T>\lambda)$}

In order to verify the adequacy of our solution, we perform calculations with $\sigma, T$, and $\epsilon(\omega)$ equal to those used in the experiments in Ref. $9(T=4.69 \lambda, \sigma=1.86 \lambda, \epsilon=1.991)$. In Fig. 3 the mean reflected intensity, for both $s$ and $p$ polarization, versus the observation angle $\theta$ above the surface is plotted for three different angles of incidence. The shape of the experimental curves (see Fig. 3 of Ref. 9) is fairly well reproduced (apart from a normalization factor) in Fig. 3, although there are some quantitative discrepancies in the shoulders of these curves that are probably due to the finite record $L$ and the differences in estimation of $\sigma$ and $T$ in theory and experiment. The authors of Refs. 8 and 38 have also obtained similar results, supporting our observations above that there is no appreciable difference between the assumption of an incident plane wave and that of a Gaussian beam. As seen in Fig. 3, there is a clear difference between the angular distribution of scattered intensity under $s$ and $p$ polarizations; whereas the former is more concentrated toward the specular direction, the latter varies less markedly with the angle of incidence and it is skewed toward the backscattering direction.

In this regime of correlation length, another value of $T$ has been analyzed $(T=3.16 \lambda)$. The rms deviation $\sigma$ takes on two different values $(\sigma=0.5 \lambda$ and $\sigma=1.9 \lambda)$, and the dielectric permittivity is raised artificially from 2.04 to $7.5^{8}$ Figure 4 shows the mean reflected intensity at incidence $\theta_{o}=0^{\circ}, 10^{\circ}$ from two dielectric interfaces $(\epsilon=$ 2.04 and $\epsilon=7.5$ ) with the same roughness ( $\sigma=1.9 \lambda$ and $T=3.16 \lambda$ ). A peak in the retroreflection direction appears for the greatest value of $\epsilon$, being larger for $s$ polarization. No backscattering peak is obtained for $\epsilon=2.04$ even though the roughness is exactly the same. This is in agreement with the results displayed in Refs. 7 and 8, in which an incident Gaussian beam stands for the incident field. As we discuss in greater detail in Section 4, the appearance of the backscattering peak for $\epsilon=7.5$ is due to the existence of multiple scattering caused by the higher reflectivity of this surface.

On the other hand, the mean transmitted intensity below the surface versus the angle of observation $\theta_{t}$ reveals a new effect of light transmission that has been satisfactorily confirmed by recent measurements ${ }^{19}$; this effect is seen in Fig. 5 for $\epsilon=2.04(\sigma=1.9 \lambda$ and $T=3.16 \lambda)$ : The mean transmitted intensity is concentrated and ex-

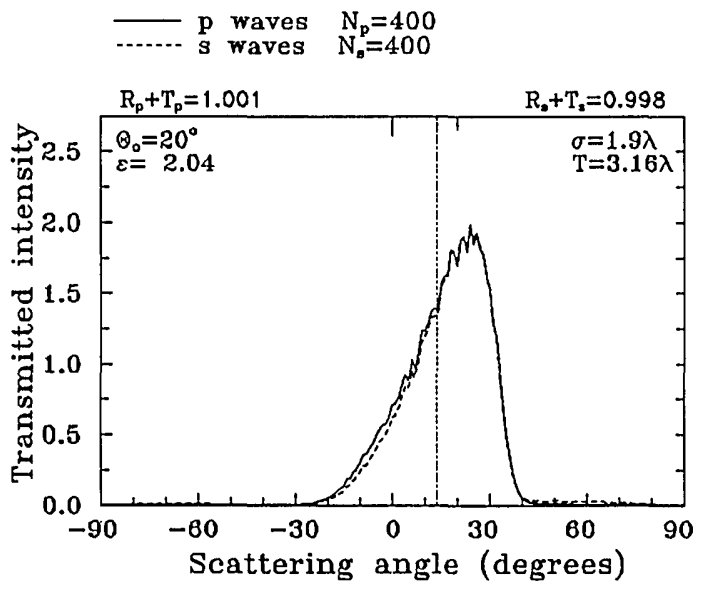

(a)
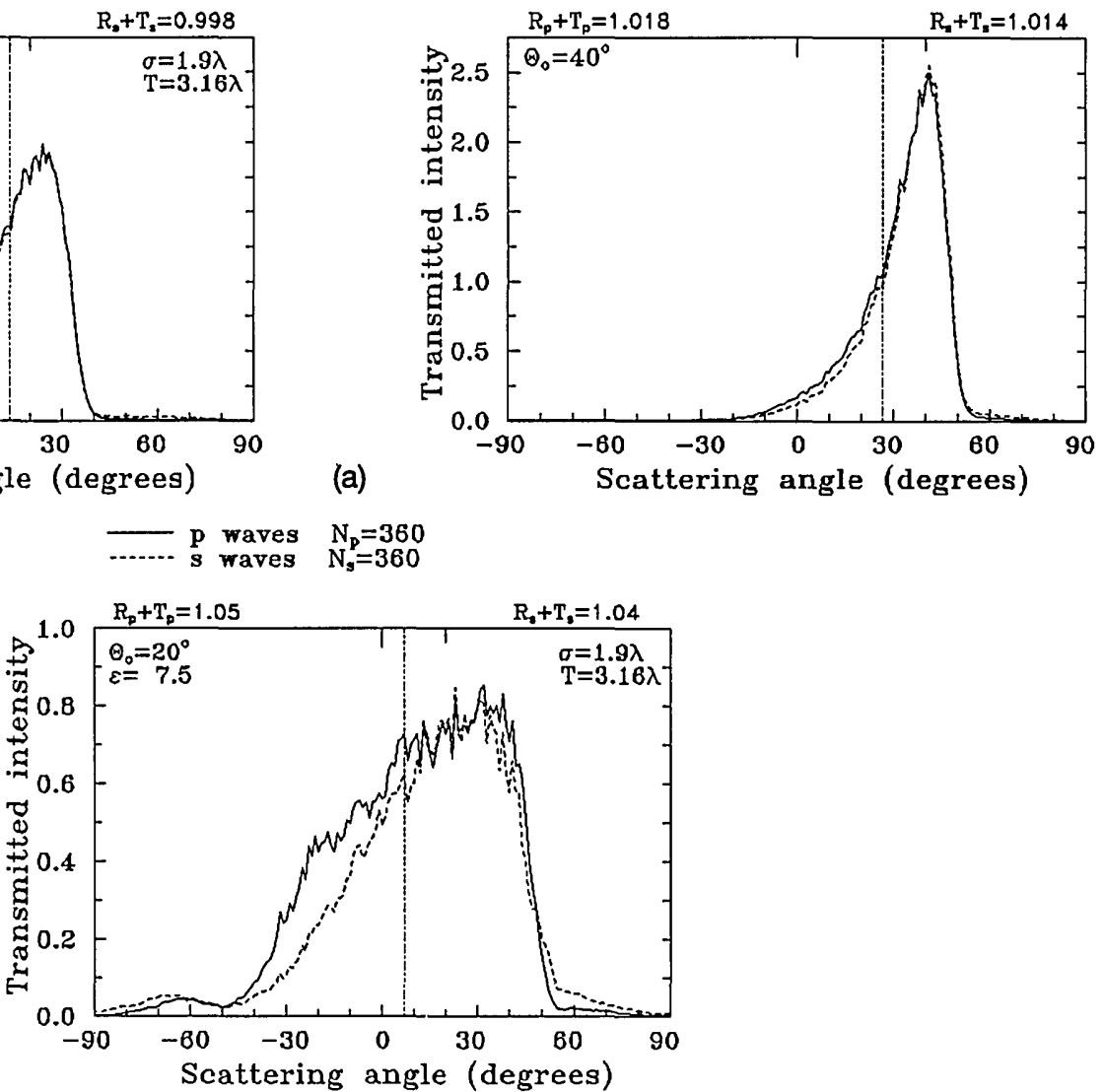

(b)

Fig. 5. Angular distribution of mean transmitted intensity from a dielectric surface with $\sigma=1.9 \lambda, T=3.16 \lambda$, (dashed curves, $s$ polarization; solid curves, $p$ polarization). The average is over 400 samples. The straight-through direction is shown by the mark at the upper right. The specular direction of refraction, namely, that from Snell's law for a plane, is marked by vertical lines: (a) $\epsilon=2.04$ at $\theta_{o}=$ $20^{\circ}, 40^{\circ}$, (b) $\epsilon=7.5$ at $\theta_{o}=20^{\circ}$. 

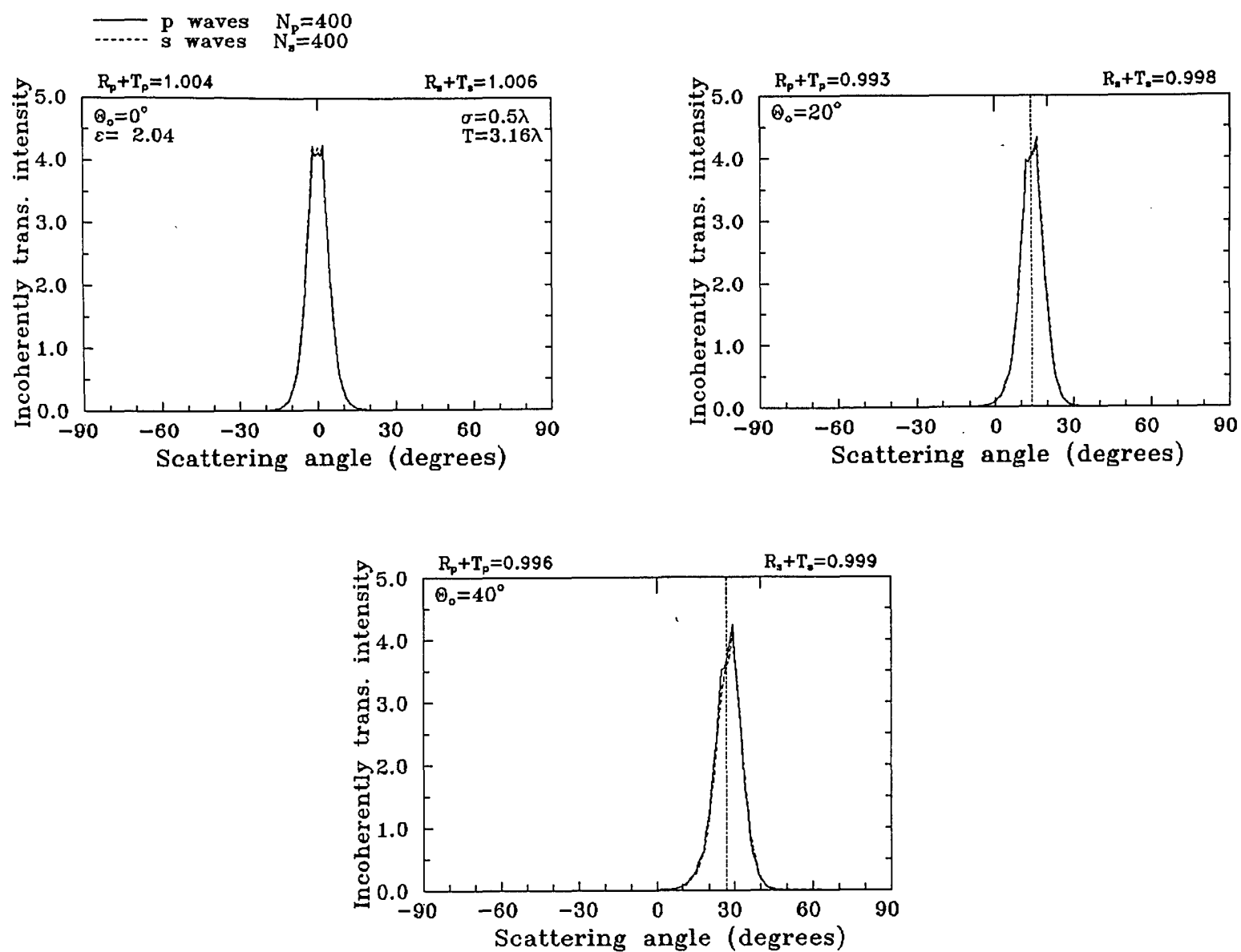

Fig. 6. Same as Fig. 5 for the diffuse component of mean transmitted (trans.) intensity: $\sigma=0.5 \lambda, T=3.16 \lambda, \epsilon=2.04 ; \theta_{o}=0^{\circ}, 20^{\circ}, 40^{\circ}$.

hibits a peak at an observation angle $\theta_{t}{ }^{\circ}$ greater than the angle given by Snell's law of refraction over the mean plane $z=0$ (dotted vertical line). $\theta_{t}{ }^{\circ}$ increases with $\theta_{o}$, and it can be equal to or even larger than the straightthrough angle $\theta_{o}$ (the little mark at the upper right of Fig. 5). While the distribution is narrow for $\epsilon=2.04$, it becomes wider for $\epsilon=7.5$. This point is further analyzed in Section 4.

If we decrease the rms deviation $(\sigma=0.5 \lambda)$, the coherent part of the distribution of transmitted light produces a large peak at the specular angle of refraction, namely, that given by the aforementioned Snell law, and the remaining diffuse component narrowly stretches around this direction (see Fig. 6). The distribution of reflected light (Fig. 7), which has no appreciable coherent contribution, resembles a Gaussian function with its maximum orientated toward the specular angle. The shape of this distribution reminds us of the analytical solution of the $\mathrm{KA}$ for perfect conductors ${ }^{6}$; in fact, as is discussed in Section 4, the $\mathrm{KA}$ is valid in this case.

It is interesting to study the influence of roughness when light incides at the Brewster angle $\left(\theta_{o}=\arctan \sqrt{\epsilon}\right)$ over the mean plane $z=0$. In Fig. 8 the mean reflected intensities for $\theta_{o}=\arctan \sqrt{2.04}=55^{\circ}$ and $T=3.16 \lambda$ are represented for both $\sigma=0.5 \lambda$ and $\sigma=1.9 \lambda$. The distribution of reflected light for $p$ polarization, $\sigma$ being $0.5 \lambda$, although significantly much smaller than that for $s$ polarization, proves to be nonnegligible. Therefore, owing to the roughness, no total transmission takes place at this angle under $p$ polarization, but remarkable differences between $s$ and $p$ polarizations still arise. Notice that no evidence of the Brewster angle is found for larger $\sigma$ [the numerical calculation of Fig. 8(b), however, fails to yield accurate results for $\theta_{o}$ larger than $50^{\circ}$ ]. The reflectance versus the angle of incidence is shown in Fig. 9 for $\sigma=0$ (plane surface), $\sigma=0.5 \lambda$, and $\sigma=1.9 \lambda(T=3.16 \lambda$ and $\epsilon=2.04)$. The zero in the reflectance obtained under $p$ polarization for a plane surface at the Brewster angle becomes a nonzero minimum when $\sigma$ is increased to $\sigma=0.5 \lambda$, which finally disappears for large roughness $(\sigma=1.9 \lambda)$. Also note the increase in the reflectance for $s$ polarization as the roughness grows at lower angles of incidence and the opposite effect at larger values of $\theta_{o}$ manifested by the crossover of the curves. As seen in Fig. 9, for $p$ polarization this crossover of reflectances makes the variation with $\sigma$ and $\theta_{o}$ more complicated. If the plane surface were infinite, there should be no distinction between $s$ and $p$ polarization in the reflectance under normal incidence (see the Fresnel coefficients, Ref. 35). Since we use our method also to obtain the reflectance from a plane surface, the finite length of the surface profiles produces an edge effect that makes both reflectances at normal incidence noncoincident. However, this difference is within the range of the numerical error.

\section{Subwavelength Correlation Length $(T<\lambda)$}

Here the surface parameters $\sigma=T=0.2 \lambda(\epsilon=2.04)$ have been investigated. In spite of the great ratio $\sigma / T$, the unitarity condition displays a negligible error (less than $2 \%$ ), and transmission takes place predominantly at the 
specular angle (Snell's law). If we subtract this strong coherent part, which is equal for both polarizations, from the total transmitted intensity, a diffuse part is obtained (Fig. 10) that is broader for $s$ than for $p$ polarization. Unlike the transmitted field, the reflected wave shows qualitative differences between both polarizations. Figure 11 illustrates this point. The mean incoherently reflected intensities resemble those calculated for perfect conductors (cf. Fig. 4 of Ref. 6); namely, it exhibits a smooth skewness toward the backscattering direction for $p$ polarization but not for $s$ polarization. The specular peak grows with increasing angle of incidence. As is evident from the reflectance (Fig. 12), the Brewster angle $\left(\theta_{o}=\right.$ $55^{\circ}$ ), which does exist for a plane, does not define for this roughness an angle of incidence with lack of reflected light for $p$ polarization. Of course, if $\sigma$ were gradually lowered, the reflectance for $p$ polarization at incidence $\theta_{o}=55^{\circ}$ would decrease from its actual value to zero for $\sigma=0$; note the curves for a plane surface, i.e., the Fresnel coefficients in Fig. 12.

\section{B. Results from the Kirchhoff Approximation}

The KA gives an analytical solution to the scattering problem for perfectly conducting surfaces. When a finite dielectric constant is considered, owing to the Fresnel coefficient's dependence on the surface coordinates, numerical computation is needed. We have outlined this $\mathrm{KA}$ numerical solution in Section 2, given by introducing Eqs. (59)-(62) into Eqs. (57) and (58). Expressions for the reflectance [Eq. (31)] and the transmittance [Eq. (32)] are also obtained. From both equations, the unitarity condition [Eq. (33)] is also calculated so we can check the accuracy of this approach.

It is well known that, since the KA considers specular reflection at the local tangent plane, it takes into account only single scattering. Furthermore, for this approximation one assumes that each scattering event occurs in a precise manner, with the use of the Fresnel coefficients. This idea can be understood in mathematical terms by looking at the equations obtained in Section 2: The KA constitutes a first-order solution to the exact equations [Eqs. (18a) and (20b) or Eqs. (36a) and (37b)]. In addition, the fields and their derivatives at each point of the surface are worked out from the corresponding Fresnel coefficients [see Eqs. (43) and (44)]. By virtue of the aforementioned reasons, one would expect that, as in a conductor, the surface should have a radius of curvature large compared with the wavelength for the plane-tangent approach involved in the KA to be reliable. However, if $\epsilon$ is small, single scattering may be also observed with more independence of $T$ and $\sigma$. This effect occurs because little radiation is reflected back after the first hit so as to permit a second scattering event. We shall see this effect in what follows.

For a comparison with the ET solutions (Figs. 6 and 7), we have chosen $\sigma=0.5 \lambda, T=3.16 \lambda$, and $\epsilon=2.04$. Figures 13 and 14 show the mean incoherently transmitted intensity and the mean reflected intensity, respectively. The agreement, as expected, is excellent, the error being less than $2 \%$ for angles of incidence below $60^{\circ}$ (at incidence
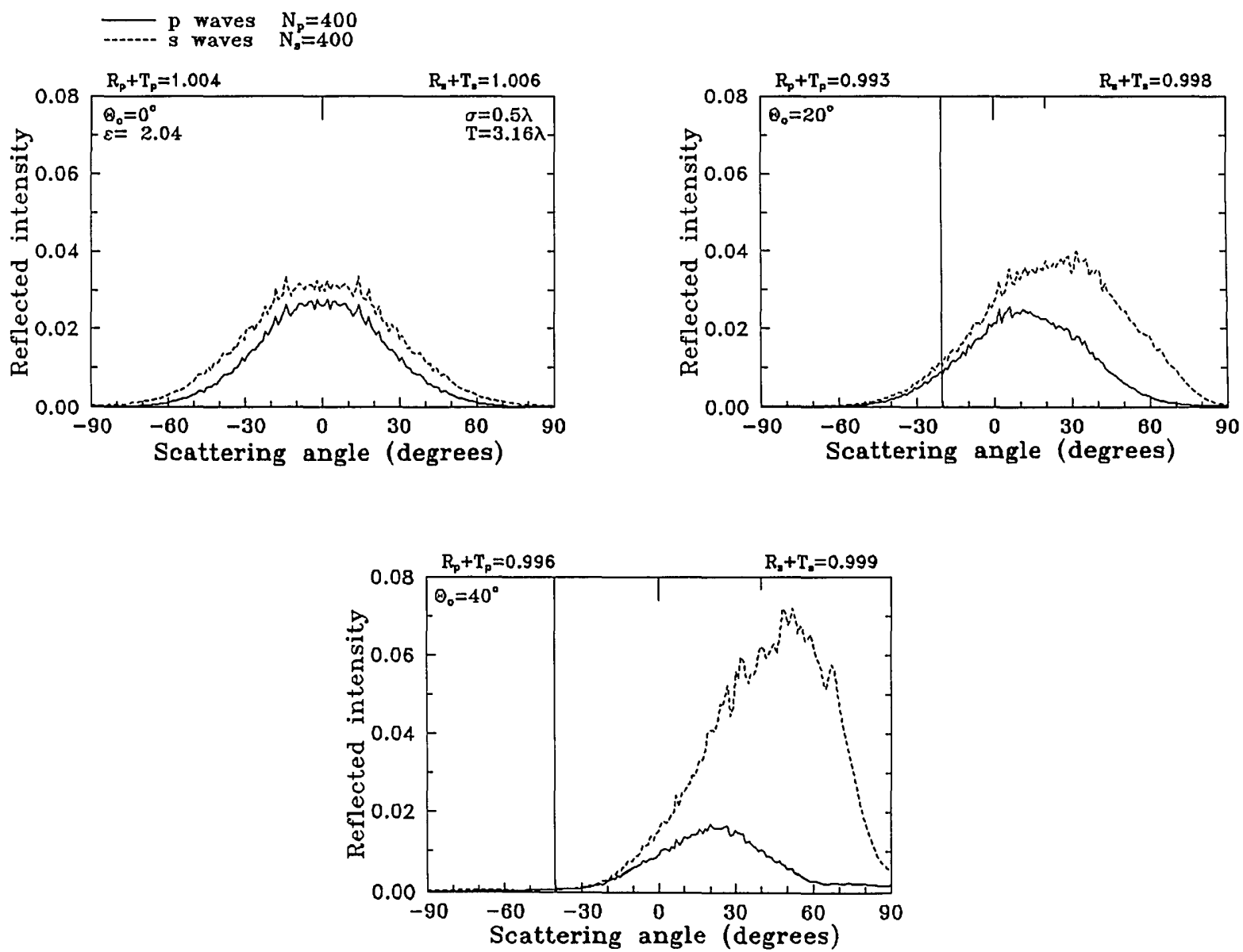

Fig. 7. Same as Fig. 3: $\sigma=0.5 \lambda, T=3.16 \lambda, \epsilon=2.04 ; \theta_{o}=0^{\circ}, 20^{\circ}, 40^{\circ}$. 


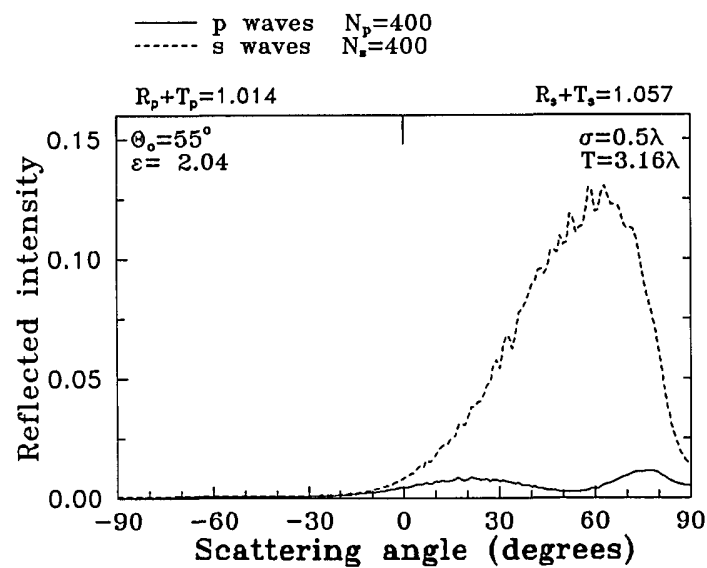

(a)

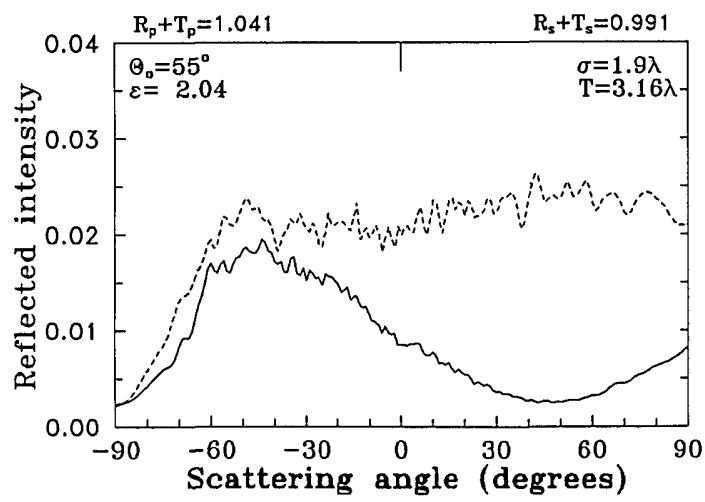

(b)

Fig. 8. Same as Fig. 3 for $T=3.16 \lambda, \epsilon=2.04$, and $\theta_{o}=55^{\circ}$ (Brewster angle for a plane): (a) $\sigma=0.5 \lambda$, (b) $\sigma=1.9 \lambda$.

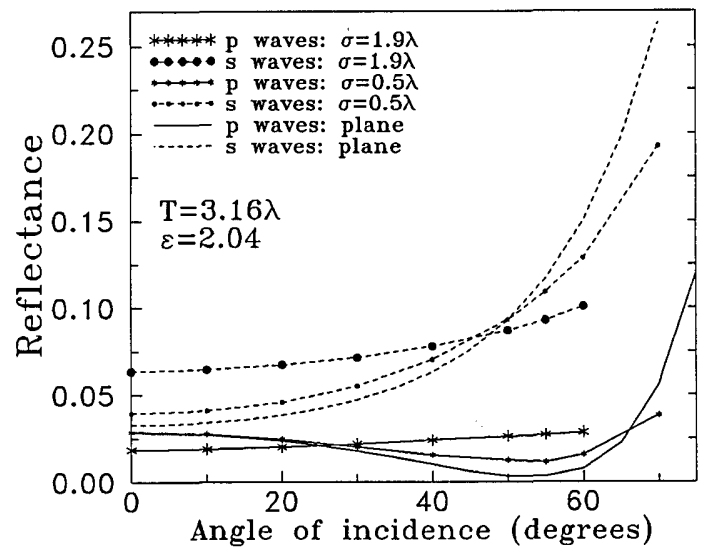

Fig. 9. Average reflectance from 400 samples versus $\theta_{o}$, for $\epsilon=$ $2.04, T=3.16 \lambda, \sigma=0.5 \lambda$, and $\sigma=1.9 \lambda$. The reflectance from a plane is also shown.

of $60^{\circ}$ or greater, the unitarity condition of the KA result fails abruptly, indicating the inaccuracy of this solution). The effect in transmission reported in Ref. 19 for large roughness $(\sigma=1.86 \lambda, T=4.69 \lambda$, and $\epsilon=1.991)$, according to which the angular distribution of transmitted light is concentrated at angles closer to the forward direction than what is predicted by Snell's law in a plane interface, is reproduced by means of the KA, as shown in Fig. 15. On the other hand, Fig. 16 includes the mean reflected intensity, which can be directly compared with the ET plot (Fig. 3) and the experimental measures ${ }^{9}$; the approxi- mation drastically fails to be valid above $40^{\circ}$ incidence and at angles of observation larger than $50^{\circ}$.

For $T<\lambda$, as for conductors, the value of $\sigma$ requires $\sigma \ll T$ for the KA to work. For instance, for $\sigma=T=$ $0.2 \lambda$ and $\epsilon=2.04$, the KA completely fails for reflected waves. Concerning transmission, only the case of $p$ polarization approaches the correct result for $\theta_{o}<20^{\circ}$.

\section{DISCUSSION}

We have seen that the phenomenon, theoretically predicted and experimentally observed,$^{19}$ of light transmission through rough dielectric surfaces around the forward direction, is also obtained by means of the KA (Fig. 15). This effect does not appear for surfaces with small correlation lengths (see Fig. 10). It can, however, be interpreted within the geometrical-optics approach, even though this is only an approximate way of explaining the scattering from surfaces whose roughness is greater than the wavelength. According to this picture, although light incides at a certain angle $\theta_{o}$ with respect to the $z$ direction, the local angle of incidence [ $\vartheta(x)$ in Fig. 2] is smaller than the global angle $\theta_{o}$ for most of the illuminated surface points, $\vartheta(x)$ thus being even zero or negative. The local Fresnel coefficients and Snell's law thus give a refraction at each sampling point much closer to the forward direction than in the plane interface case. The relatively small dielectric constant ensures that light is not reflected back after the first hit with the interface and thus
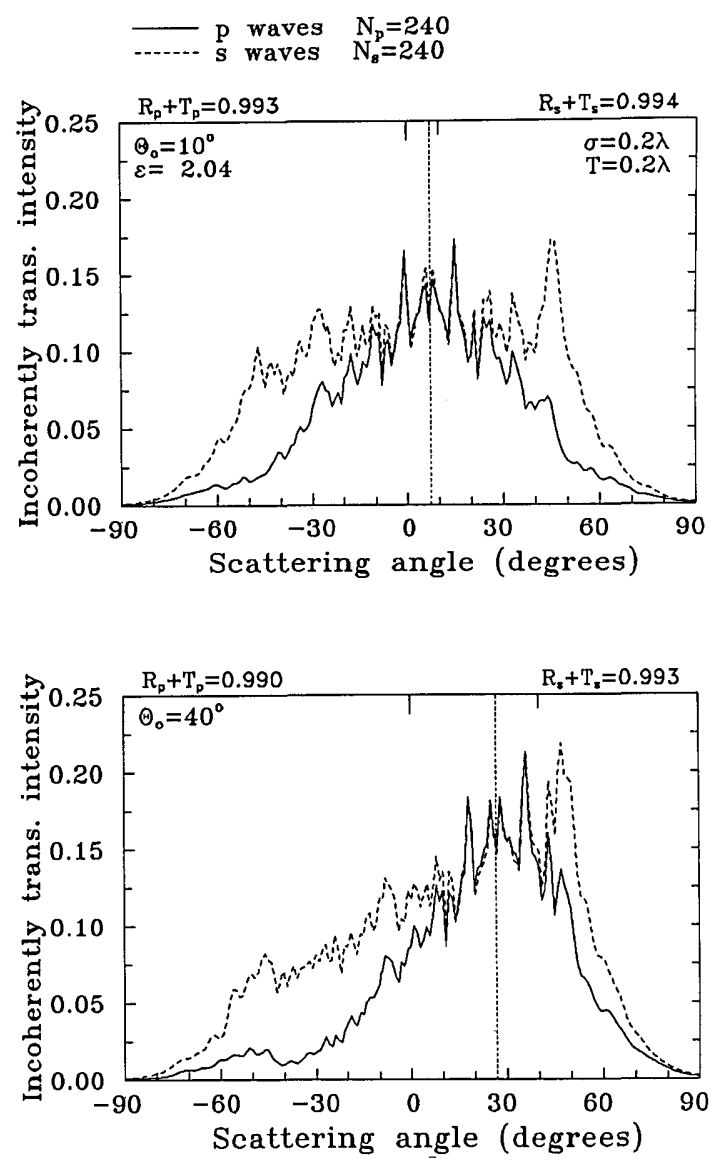

Fig. 10. Same as Fig. 5 for the diffuse component of mean transmitted (trans.) intensity for $\sigma=0.2 \lambda, T=0.2 \lambda$, and $\epsilon=2.04$; $\theta_{o}=10^{\circ}, 40^{\circ}$. 

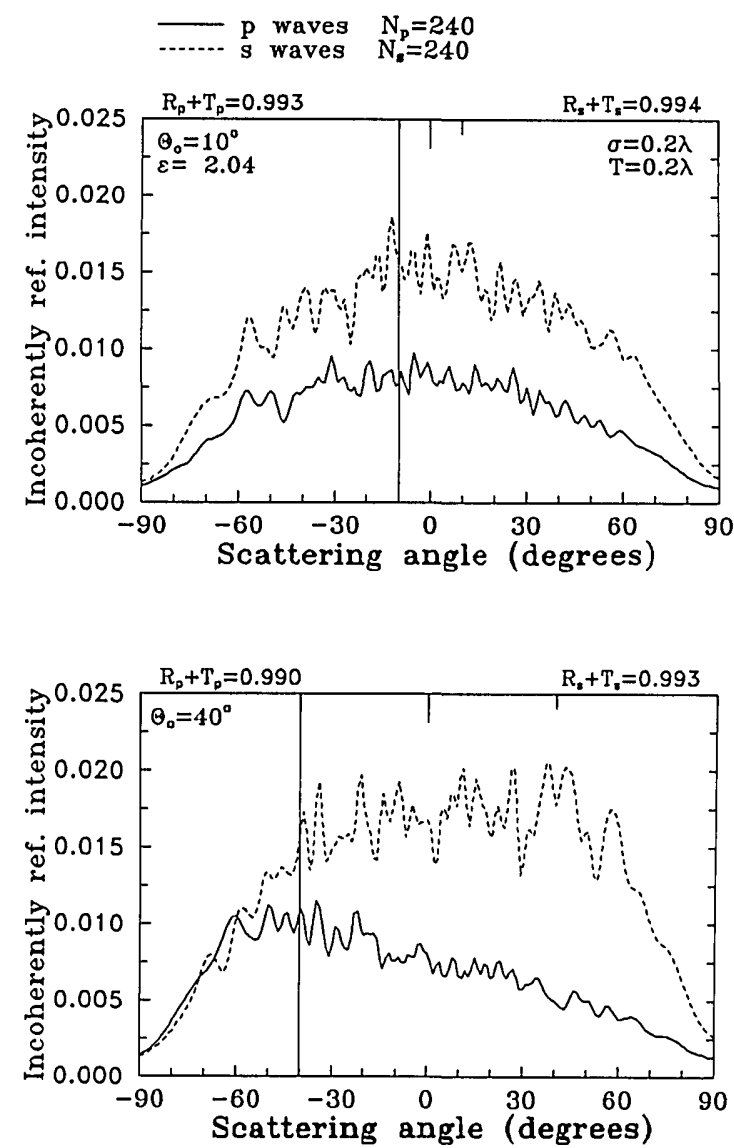

Fig. 11. Same as Fig. 3 for the diffuse component of mean reflected (ref.) intensity for $\sigma=0.2 \lambda, T=0.2 \lambda$, and $\epsilon=2.04$; $\theta_{o}=10^{\circ}, 40^{\circ}$.

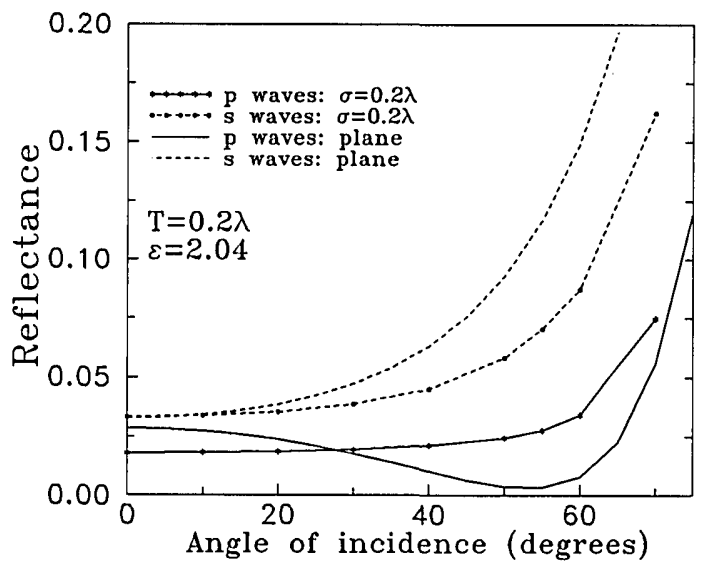

Fig. 12. Same as Fig. 9 from 240 sämples versus $\theta_{o}$, for $\sigma=$ $0.2 \lambda, T=0.2 \lambda$, and $\epsilon=2.04$. The reflectance from a plane is also shown.

is not multiply scattered, since the contribution of the waves reflected from single scattering that could be scattered again is negligible (see the Fresnel coefficients in Fig. 9). Adding all these particular rays, we obtain a narrow distribution centered at an angle of transmission larger than the one predicted by Snell's law for a plane surface $z=0$. This phenomenon, which is due to single scattering and high slope, is possible only in surfaces with high transmissivity (low $\epsilon$ ) and $T>\lambda$. Large rms deviation $\sigma$ is also required for this effect to be appreciable; for instance, $\sigma=0.5 \lambda$ (see Fig. 6) is not large enough.
By artificially raising $\epsilon$, we increase the reflectivity; hence double-scattering events take place, and this produces a broadening of the distribution of transmitted light, which no longer peaks in a precise direction (see Fig. 5 for $\epsilon=7.5$ ). This higher reflectivity at larger $\epsilon$ also explains the backscattering peak observed within this regime of $\sigma$ and $T$ for perfect conductors, ${ }^{5,6,20}$ real metals, ${ }^{3,4,7-10}$ and dielectrics ${ }^{8}$ with high $\epsilon$. The diagrammatic approach of Refs. 3, 4, and 20 describes how the backscattering phenomenon can be understood in terms of double or multiple scattering. The results presented above (Fig. 4) show a backscattering peak for a dielectric constant large enough to enhance doubly scattered reflected energy. As a matter of fact, since the reflectance is considerably smaller for $p$ waves than for $s$ waves, the peak is lower for $p$ polarization. Therefore we can infer that the broadening of the distribution of transmitted light is intimately related to the enhancement in the retroreflection direction of the distribution of reflected light. Both effects depend on double- or multiple-scattering processes. Of course, we are referring to surfaces with large correlation length and large roughness but $\sigma \leq T$. For real metals and perfect conductors, the much larger reflectance gives rise to a much higher peak of backscattering, which is almost equal for both polarizations.

Keeping the geometrical-optics picture in mind, we may easily understand the disappearance of the Brewster effect: Surface roughness implies that the local angle of incidence takes on a range of values about the overall angle of incidence under consideration. As a consequence, even though light is incident at the Brewster angle with respect to the average plane, e.g., $\theta_{o}=55^{\circ}$ in Fig. 9, light is actually reflected at many points of the surface according to the corresponding local angle of incidence. This effect becomes more apparent as $\sigma$ increases (see Fig. 8). The reflectance curves (Fig. 9) support this explanation. We can qualitatively account for the behavior of the reflectance at each $\theta_{o}$ by averaging the Fresnel coefficients over a certain interval of angles around $\theta_{o}$; the rougher the surface, the wider the interval. This also explains why for lower angles of incidence the reflectance for $\sigma=1.9 \lambda$ is greater than that for $\sigma=0.5 \lambda$.

If the correlation length is smaller than the wavelength, several valleys and hills may be included within one wavelength. Therefore scattering cannot be explained as a local interaction between rays and plane pieces of surface. Perturbation techniques ${ }^{24-31}$ have been widely applied for this regime. Although the mathematical series thus obtained converges only when $\sigma \ll \mathrm{T}$, the physical interpretation involved in these methods is useful. The surface roughness produces a decrease of the specular peak and a consequent increase of the diffuse component in both reflection and transmission. These effects increase with $\sigma$ and decrease with $\theta_{o}$ (see Figs. 10 and 11). The qualitative behavior of the mean reflected intensity (Fig. 11) does not differ too much from that for perfect conductors, ${ }^{5,6}$ except for the $p$-polarized specular peak not observed for metallic surfaces.

Finally, we comment on the range of validity of the KA. It has been clearly demonstrated that the criterion shown in Ref. 6, established for perfect conductors, is too restrictive for transmission in dielectric surfaces. The result with $\sigma=1.86 \lambda, T=4.69 \lambda$, and $\theta_{o}=40^{\circ}\left(\sigma / T \cos \theta_{o}=\right.$ 

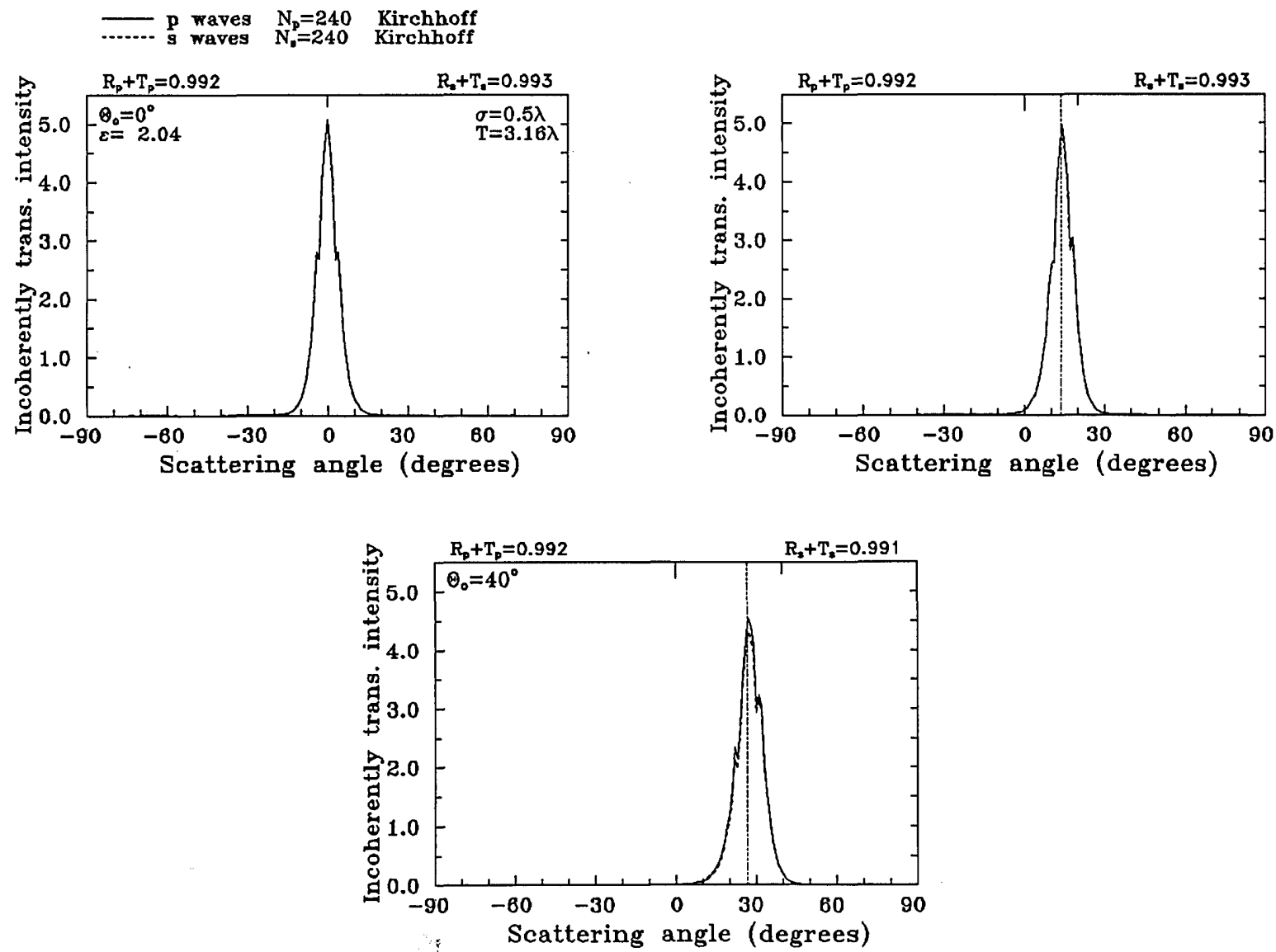

Fig. 13. Same as Fig. 6 for mean transmitted (trans.) intensity KA.
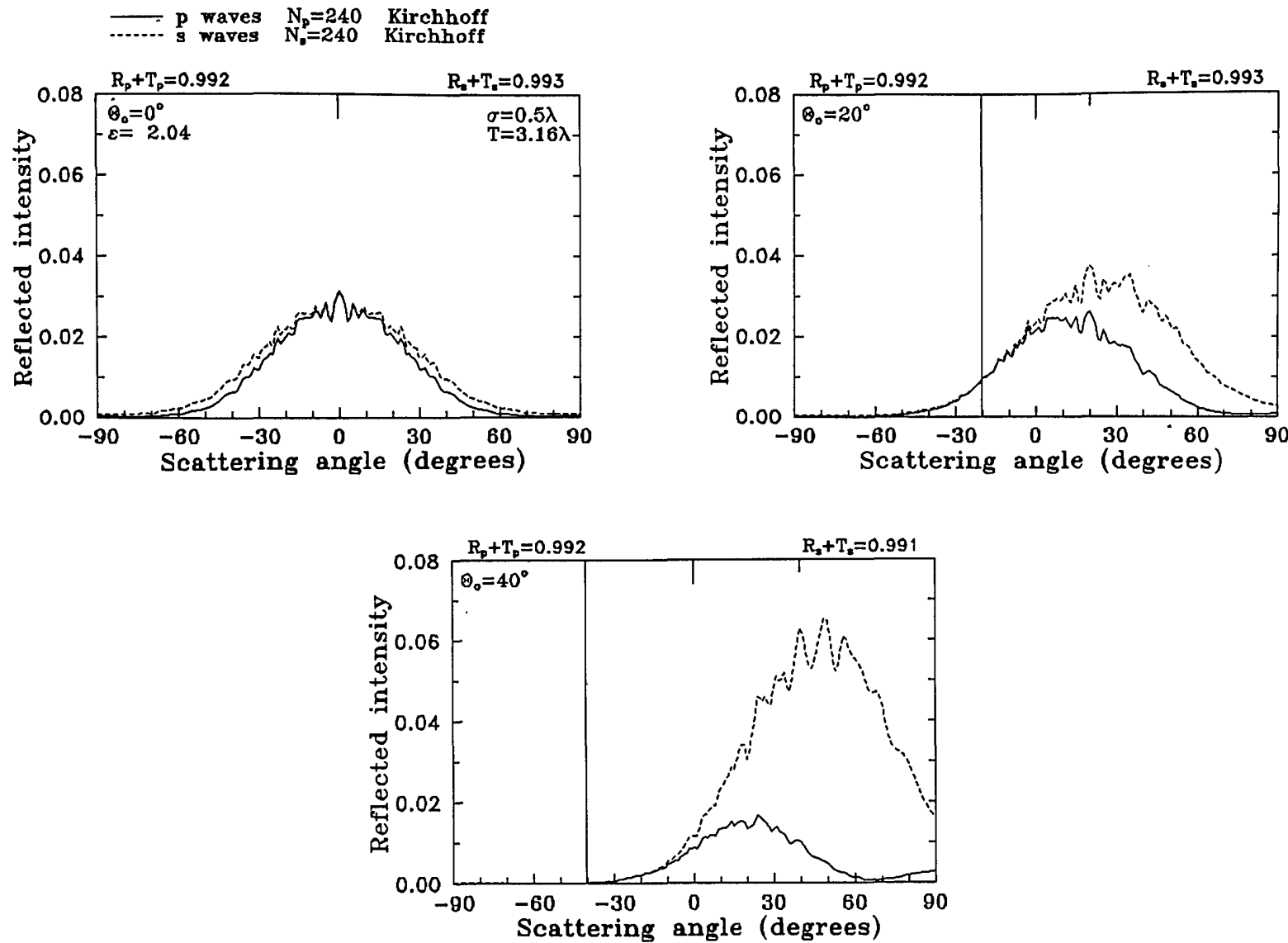

Fig. 14. Same as Fig. 7 with the KA. 

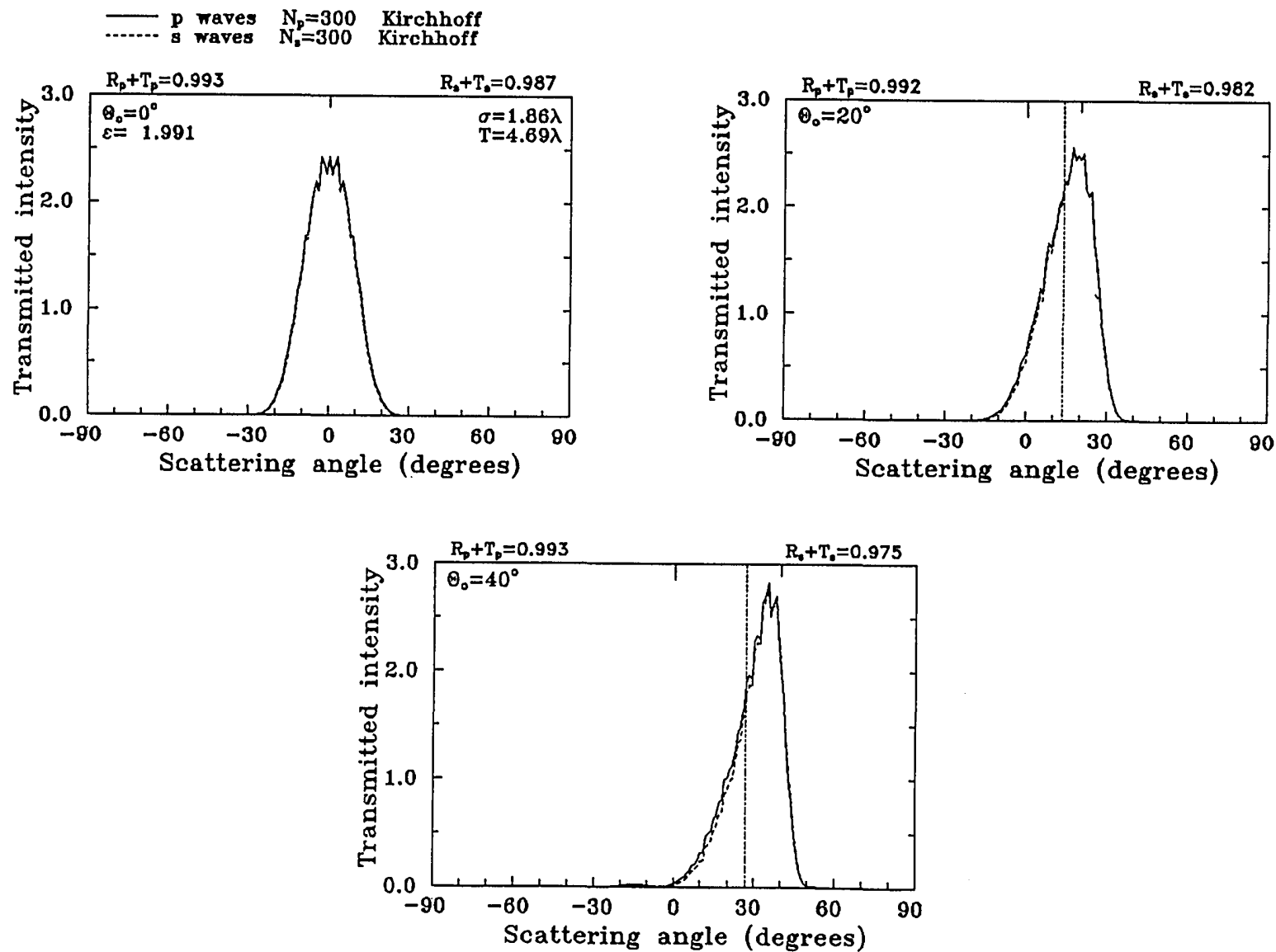

Fig. 15. Same as Fig. 5 for $\sigma=1.86 \lambda, T=4.69 \lambda$, and $\epsilon=1.991$ at $\theta_{0}=0^{\circ}, 20^{\circ}, 40^{\circ}$ with the KA.
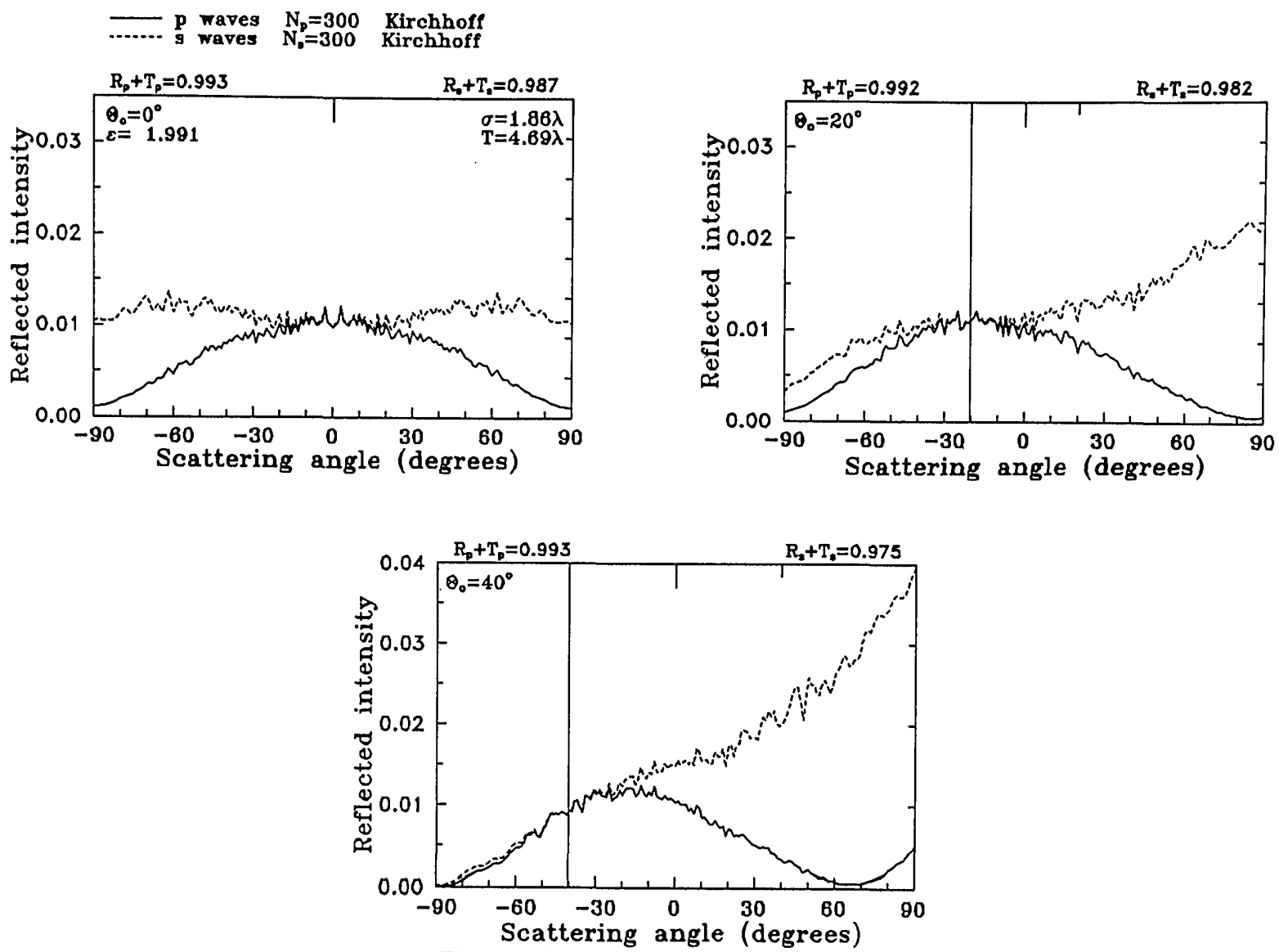

Fig. 16. Same as Fig. 3 with the KA. 
0.5) from Fig. 15 is similar to the ET solution of Fig. 5, although $\epsilon$ is slightly different in these figures (we have plotted only a few of our computer results; when $\epsilon$ is the same as in Fig. 15, the coincidence with the KA is total). Hence the criterion depends on the dielectric constant too. The relevant point is the relative importance of the multiple-scattering contribution. Thus, if $\epsilon$ ensures that the largest part of the incident energy is transmitted through the surface after a single scattering event, multiple scattering will hardly contribute to the mean intensities, even though the surface is very rough $(\sigma \leq T)$. It should be remarked, however, that, for reflection, although the criterion of Ref. 6 is still restrictive for dielectrics, the KA yields worse results than those for transmission at larger angles of incidence and observation, as depicted in Fig. 16.

\section{CONCLUSIONS}

From the results obtained in this paper, some important conclusions can be drawn concerning the scattering of light and other electromagnetic waves from rough random dielectric surfaces:

(1) The anomalous refraction encountered for $T>\lambda$ and large $\sigma$ is due to single scattering. For this reason, the KA yields a good account of this effect.

(2) The broadening of the distribution of transmitted light for $T>\lambda$ when $\epsilon$ is increased (the reflectance then growing with $\epsilon$ ) is due to double- and higher-order scattering events. The backscattering peak appearing in the mean reflected intensity is also produced by multiple scattering. The larger reflectance of the $s$-polarized waves implies a stronger enhancement in the retroreflection direction for this polarization. Therefore this enhanced backscattering appears in the same surfaces in which the broadening of the transmitted light distribution occurs.

(3) The Brewster effect disappears as $\sigma$ is gradually increased. The reason is the net contribution from points on the surface at which light is locally incident at angles different from this overall Brewster angle. Curves showing the reflectance (Fig. 9) versus the angle of incidence demonstrate this point. The same argument explains why the reflectance increases with $\sigma$.

(4) The range of validity of the $\mathrm{KA}$ is broader for dielectrics than for perfect conductors. Also, this adequacy of the KA for dielectrics is much wider for transmission than for reflection. Since the dielectric transmits practically all the incident energy in the first scattering events, the contribution from multiple scattering is almost negligible even for relatively high roughness parameters. However, like that in perfect conductors, the KA fails at $T<\lambda$ unless $\sigma \ll T$.

(5) Surfaces with small $T$ produce a diffusely transmitted component that increases with $\sigma$ values; the specular peak decreases.

The numerical method used here, based on the ET, thus is revealed as a useful tool to solve the scattering from rough random dielectric surfaces.

Finally, we note the recent paper ${ }^{38}$ by Saillard and Maystre, which addresses some aspects dealt with here on the reflectivity, including results similar to those of Fig. 3.

\section{ACKNOWLEDGMENTS}

This research was supported by the Comisión Interministerial de Ciencia y Tecnología under grant PB0278. J. A. Sánchez-Gil acknowledges a grant from Ministerio de Educación y Ciencia. The authors thank J. M. SotoCrespo for his help during the first stages of this research. Discussions with J. C. Dainty and A. A. Maradudin through a NATO travel grant are appreciated.

\section{REFERENCES}

1. V. Celli, A. A. Maradudin, A. M. Marvin, and A. R. McGurn, "Some aspects of light scattering from a randomly rough metal surface," J. Opt. Soc. Am. A 2, 2225-2239 (1985).

2. P. Tran and V. Celli, "Monte Carlo calculation of backscattering enhancement for a randomly rough grating," J. Opt. Soc. Am. A 5, 1635-1637 (1988).

3. E. R. Méndez and K. A. O'Donnell, "Observation of depolarization and backscattering enhancement in light scattering from Gaussian random surfaces," Opt. Commun. 61, 91-95 (1987).

4. K. A. O'Donnell and E. R. Méndez, "Experimental study of scattering from characterized random surfaces," J. Opt. Soc. Am. A 4, 1194-1205 (1987).

5. M. Nieto-Vesperinas and J. M. Soto-Crespo, "Monte Carlo simulations for scattering of electromagnetic waves from perfectly conductive random rough surfaces," Opt. Lett. 12, 979-981 (1987).

6. J. M. Soto-Crespo and M. Nieto-Vesperinas, "Electromagnetic scattering from very rough random surfaces and deep reflection gratings," J. Opt. Soc. Am. A 6, 367-384 (1989).

7. A. A. Maradudin, E. R. Méndez, and T. Michel, "Backscattering effects in the elastic scattering of $p$-polarized light from a large amplitude random metallic grating," Opt. Lett. 14, 151-153 (1989).

8. A. A. Maradudin, T. Michel, A. R. McGurn, and E. R. Méndez, Ann. Phys. (NY) 203, 255-307 (1990).

9. A. J. Sant, J. C. Dainty, and M. J. Kim, "Comparison of surface scattering between identical, randomly rough metal and dielectric diffusers," Opt. Lett. 14, 1183-1185 (1989).

10. M. J. Kim, J. C. Dainty, A. T. Friberg, and A. J. Sant, "Experimental study of enhanced backscattering from one- and two-dimensional random rough surfaces," J. Opt. Soc. Am A 7, 569-577 (1990).

11. M. Nieto-Vesperinas and J. M. Soto-Crespo, "Light-diffracted intensities from very deep gratings," Phys. Rev. B 38, 72507259 (1988)

12. M. Nieto-Vesperinas and J. M. Soto-Crespo, "Connection between blazes from gratings and enhancements from random rough surfaces," Phys. Rev. B 39, 8193-8197 (1989).

13. J. M. Soto-Crespo and M. Nieto-Vesperinas, "Enhancement of all antispecular orders from deep gratings," Opt. Commun. 69, 185-188 (1989).

14. E. I. Thorsos, "The validity of the Kirchhoff approximation for rough surface scattering using a Gaussian roughness spectrum," J. Acoust. Soc. Am. 83, 78-92 (1987).

15. E. I. Thorsos and D. R. Jackson, "The validity of the perturbation approximation for rough surface scattering using a Gaussian roughness spectrum," J. Acoust. Soc. Am. 86, 261277 (1989).

16. M. Nieto-Vesperinas and J. M. Soto-Crespo, "Monte Carlo calculations of speckle contrast from perfectly conductive random rough surfaces," Opt. Commun. 75, 215-218 (1990).

17. J. M. Soto-Crespo, M. Nieto-Vesperinas, and A. T. Friberg, "Scattering from slightly rough random surfaces: a detailed study on the validity of the small perturbation method," J. Opt. Soc. Am. A 7, 1185-1201 (1990).

18. C. Macaskill, "Geometric optics and enhanced backscatter from very rough surfaces," J. Opt. Soc. Am. A 8, 88-96 (1991).

19. M. Nieto-Vesperinas, J. A. Sánchez-Gil, A. J. Sant, and J. C. Dainty, "Light transmission from a randomly rough dielectric diffuser: theoretical and experimental results," Opt. Lett. 15, 1261-1263 (1990). 
20. M. Nieto-Vesperinas and J. C. Dainty, eds., Scattering in Volumes and Surfaces (North-Holland, Amsterdam, 1990).

21. P. Beckmann and A. Spizzichino, The Scattering of Electromagnetic Waves from Rough Surfaces (Macmillan, New York, 1963).

22. P. Beckmann, "Scattering of light by rough surfaces," in Progress in Optics VI, E. Wolf, ed. (North-Holland, Amsterdam, 1961), pp. 55-69.

23. F. G. Bass and I. M. Fuks, Wave Scattering from Statistically Rough Surfaces (Pergamon, Oxford, 1979).

24. S. O. Rice, "Reflection of electromagnetic waves from slightly rough surfaces," Commun. Pure Appl. Math. 4, 4808-4816 (1951).

25. G. R. Valenzuela, "Depolarization of electromagnetic waves by slightly rough surfaces," IEEE Trans. Antennas Propag. AP-15, 552-557 (1967)

26. V. Celli, A. Marvin, and F. Toigo, "Light scattering from rough surfaces," Phys. Rev. B 11, 1779-1786 (1975).

27. G. S. Agarwal, "Interaction of EM waves at rough dielectric surfaces," Phys. Rev. B 15, 2371-2383 (1977).

28. F. Toigo, A. Marvin, V. Celli, and N. R. Hill, "Optical properties of rough surfaces: general theory and small roughness limit," Phys. Rev. B 15, 5618-5626 (1977).

29. N. Garcia, V. Celli, and M. Nieto-Vesperinas, "Exact multiple scattering of waves from random rough surfaces," Opt. Commun. 30, 279-281 (1979).

30. M. Nieto-Vesperinas and N. Garcia, "A detailed study of the scattering of a scalar wave from random rough surfaces," Opt. Acta 28, 1651-1672 (1981).

31. M. Nieto-Vesperinas, "Depolarization of electromagnetic waves scattered from slightly rough random surfaces: a study by means of the extinction theorem," J. Opt. Soc. Am. 72, 539-547 (1982).

32. D. N. Pattanayak and E. Wolf, "General form and a new interpretation of the Ewald-Oseen extinction theorem," Opt. Commun. 6, 217-220 (1972).

33. A. K. Fung and M. F. Chen, "Numerical simulation of scattering from simple and composite random surfaces," J. Opt. Soc. Am. A 2, 2274-2284 (1985).

34. J. D. Jackson, Classical Electodinamics, 2nd ed. (Wiley, New York, 1975), Sec. I.5.

35. M. Born and E. Wolf, Principles of Optics, 6th ed. (Pergamon, Oxford, 1980), Sec. I.5.

36. M. Cadilhac, in Electromagnetic Theory of Gratings, R. Petit, ed., Vol. 22 of Topics in Current Physics (SpringerVerlag, Berlin, 1980), p. 54.

37. M. Abramowitz and I. A. Stegun, Handbook of Mathematical Functions (Dover, New York, 1965), p. 364.

38. M. Saillard and D. Maystre, "Scattering from metallic and dielectric surfaces," J. Opt. Soc. Am. A 7, 982-990 (1990); see also M. Saillard, "Theoretical and numerical study of light scattering from dielectric and conducting rough surfaces," Ph.D. dissertation (University of Aix-Marseille III, Aix-enProvence, France, 1990). 